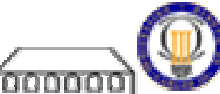

Working Paper 06-30

Statistics and Econometrics Series 12

May, 2006
Departamento de Estadística Universidad Carlos III de Madrid

Calle Madrid, 126

28903 Getafe (Spain)

Fax (34) 91 624-98-49

\title{
ON THE CONCEPT OF DEPTH FOR FUNCTIONAL DATA
}

\author{
Sara López-Pintado and Juan Romo*
}

\begin{abstract}
The statistical analysis of functional data is a growing need in many research areas. We propose a new depth notion for functional observations based on the graphic representation of the curves. Given a collection of functions, it allows to establish the centrality of a function and provides a natural center-outward ordering of the sample curves. Robust statistics such as the median function or a trimmed mean function can be defined from this depth definition. Its finite-dimensional version provides a new depth for multivariate data that is computationally very fast and turns out to be convenient to study high-dimensional observations. The natural properties are established for the new depth and the uniform consistency of the sample depth is proved. Simulation results show that the trimmed mean presents a better behavior than the mean for contaminated models. Several real data sets are considered to illustrate this new concept of depth. Finally, we use this new depth to generalize to functions the Wilcoxon rank sum test. It allows to decide whether two groups of curves come from the same population. This functional rank test is applied to girls and boys growth curves concluding that they present different growth patterns.
\end{abstract}

Key Words: Functional data; Data depth; Rank test for functions.

*López-Pintado, Rutgers University, e-mail: saral@stat.rutgers.edu; Romo, Departamento de Estadística, Universidad Carlos III de Madrid, e-mail: juan.romo@uc3m.es. 


\title{
On the Concept of Depth for Functional Data
}

\author{
Sara López-Pintado and Juan Romo*
}

\begin{abstract}
The statistical analysis of functional data is a growing need in many research areas. We propose a new depth notion for functional observations based on the graphic representation of the curves. Given a collection of functions, it allows to establish the "centrality" of a function and provides a natural center-outward ordering of the sample curves. Robust statistics such as the median function or a trimmed mean function can be defined from this depth definition. Its finite-dimensional version provides a new depth for multivariate data that is computationally very fast and turns out to be convenient to study high-dimensional observations. The natural properties are established for the new depth and the uniform consistency of the sample depth is proved. Simulation results show that the trimmed mean presents a better behavior than the mean for contaminated models. Several real data sets are considered to illustrate this new concept of depth. Finally, we use this new depth to generalize to functions the Wilcoxon rank sum test. It allows to decide whether two groups of curves come from the same population. This functional rank test is applied to girls and boys growth curves concluding that they present different growth patterns.

KEY WORDS: Functional Data; Data Depth; Rank Test for Functions.
\end{abstract}

*Department of Statistics, Universidad Carlos III de Madrid, 28903, Getafe, Madrid, Spain (e-mail: saral@stat.rutgers.edu; juan.romo@uc3m.es). This research was supported in part by Spanish Ministry of Education and Science grants BEC2000-0167 and BEC2005-03424. 


\section{INTRODUCTION}

The data output sophistication in different research fields requires to advance in the statistical analysis of complex data. In functional data analysis, each observation is a real function $x_{i}(t), i=1, \ldots, n, t \in I$, where $I$ is an interval in $\mathbb{R}$. There are several reasons that make necessary the study of functional data. In many research areas (medicine, biology, economics, engineering), the data generating process is naturally a stochastic function. Moreover, many problems are better approached if the data are considered as functions. For instance, if each curve is observed at different points, a multivariate analysis would not be valid, and it is therefore necessary to smooth the data and treat them as continuous functions defined in a common interval.

Multivariate techniques such as principal components, analysis of variance and regression methods have already been extended to a functional context (see Ramsay and Silverman, 2005). A fundamental task in functional data analysis is to provide an ordering within a sample of curves that allows the definition of order statistics such as ranks and L-statistics. A natural tool to analyze these functional data aspects is the idea of statistical depth. It has been introduced to measure the "centrality" or the "outlyingness" of an observation with respect to a given data set or an underlying distribution. In this paper, we propose a new definition of depth for functional observations. It permits to order a sample of curves from the center-outward and to extend robust statistics to a functional context. For example, a median function is a curve with the highest depth.

The notion of depth was first considered for multivariate data to generalize order statistics, ranks and medians to higher dimensions. Given a distribution of probability $F$ in $\mathbb{R}^{d}$, a statistical depth assigns to each point $x$ a real non-negative bounded value $D(x, F)$. Some depth definitions for multivariate data have been proposed by Mahalanobis (1936), Tukey (1975), Oja (1983), Liu (1990), Singh (1991), Fraiman and Meloche (1999), Vardi and Zhang (2001) and Zuo (2003). Liu (1990) and Zuo and Serfling (2000) introduced and studied four key properties a depth should verify: affine invariance, maximality at center, monotonicity and vanishing at infinity. Data depth can be widely applied. For example, Liu and Singh (1993) presented a nonparametric multivariate rank test using a quality depth index and 
Liu (1995) proposed control charts for multivariate processes based on depth. Also, Liu, Parelius and Singh (1999) offered depth based tools for multivariate analysis; for instance, they defined trimmed regions, central regions and contours, and constructed a scale curve to visualize sample dispersion. In addition, Rousseeuw and Hubert (1999) introduced the idea of regression depth and Li and Liu (2004) designed a graphic tool and a test to check if two multivariate samples come from the same population.

The main goal of this paper is to propose a new notion of depth for functional data. It is based on the graphic representation of the functions and makes use of the bands defined by their graphs on the plane. Its finite-dimensional version is an alternative definition of depth for multivariate data, verifying essentially all the properties studied in Zuo and Serfling (2000). In addition, it has the advantage of being computationally very fast, which makes it adequate for analyzing high-dimensional data. Some asymptotic results, such as the uniform convergence of the sample depth and deepest point, are established. Most of these properties are extended to functions. With this new definition we can also generalize the concepts of multivariate $L$-estimates (in particular, trimmed means) to a functional context, where robust methods are possibly more useful than in multivariate problems, because there are more ways for outliers to affect functional statistics. For instance, a curve could be an "outlier" without having any unusually large value. Depth is particularly useful for identifying this kind of outliers; here, besides magnitude, shape is also relevant. Fraiman and Muniz (2001) introduced and studied a previous concept of depth for functional observations based on the integrals of univariate depths.

The paper is organized as follows. In section 2, we present the new definition of band depth for functional data. Section 3 explores its finite-dimensional version. In section 4 , the functional version properties are analyzed. A generalized band depth, more convenient for irregular functions, is defined in section 5. Section 6 contains some simulations illustrating the robustness of estimates based on the proposed depth. In section 7 , real data examples are discussed and used to check the band depth performance. A rank test for functions is introduced in section 8 and applied to decide whether two groups of real curves come from the same population. Finally, in section 9, we outline the main conclusions of this paper. The proofs are included in the Appendix. 


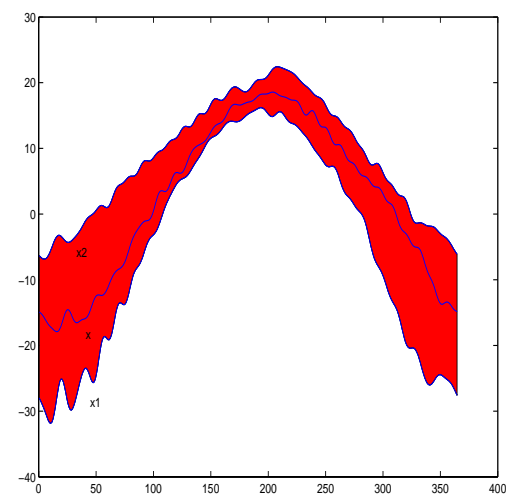

(a)

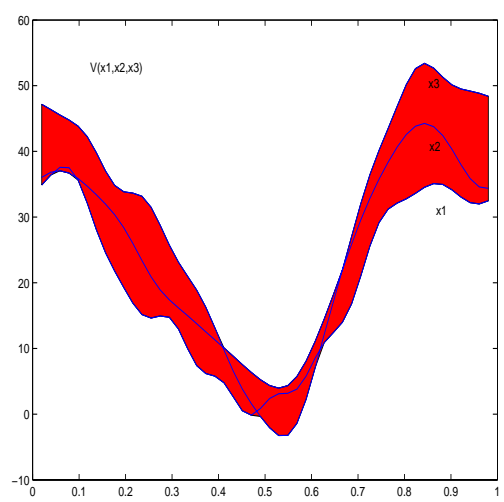

(b)

Figure 1: (a) Band defined by two curves $x_{1}, x_{2}$ and a third curve $x$ belonging to the band and (b) band determined by three curves $x_{1}, x_{2}$ and $x_{3}$.

\section{A BAND DEPTH FOR FUNCTIONAL DATA}

Our proposal follows a graph based approach. We recall definitions about function graphs that will be used throughout the paper. Let $x_{1}(t), \ldots, x_{n}(t)$ be a collection of real functions. Although the following ideas can be given for more general observations, we will restrict the exposition to functions in the space $C(I)$ of real continuous functions on the compact interval $I$. The graph of a function $x$ is the subset of the plane $G(x)=\{(t, x(t)): t \in I\}$. The band in $\mathbb{R}^{2}$ delimited by the curves $x_{i_{1}}, \ldots, x_{i_{k}}$ is

$$
\begin{aligned}
B\left(x_{i_{1}}, x_{i_{2}}, \ldots, x_{i_{k}}\right) & =\left\{(t, y): t \in I, \min _{r=1, \ldots, k} x_{i_{r}}(t) \leq y \leq \max _{r=1, \ldots, k} x_{i_{r}}(t)\right\}= \\
& =\left\{(t, y): t \in I, y=\alpha_{t} \min _{r=1, \ldots, k} x_{i_{r}}(t)+\left(1-\alpha_{t}\right) \max _{r=1, \ldots, k} x_{i_{r}}(t), \alpha_{t} \in[0,1]\right\} .
\end{aligned}
$$

Figure 1a presents the band $B\left(x_{1}, x_{2}\right)$ given by two curves; the graph of the function $x$ is included in the band. Figure $1 \mathrm{~b}$ shows the band given by three curves $B\left(x_{1}, x_{2}, x_{3}\right)$. For any function $x$ in $x_{1}, \ldots, x_{n}$, the quantity

$$
S_{n}^{(j}(x)=\left(\begin{array}{c}
n \\
j
\end{array}\right)^{-1} \sum_{1 \leq i_{1}<i_{2}<\ldots<i_{j} \leq n} I\left\{G(x) \subset B\left(x_{i_{1}}, x_{i_{2}}, \ldots, x_{i_{j}}\right)\right\}, j \geq 2,
$$

expresses the proportion of bands $B\left(x_{i_{1}}, x_{i_{2}}, \ldots, x_{i_{j}}\right)$ determined by $j$ different curves $x_{i_{1}}, x_{i_{2}}, \ldots, x_{i_{j}}$ containing the graph of $x$. (I\{A\} is one if $A$ is true and zero otherwise). 


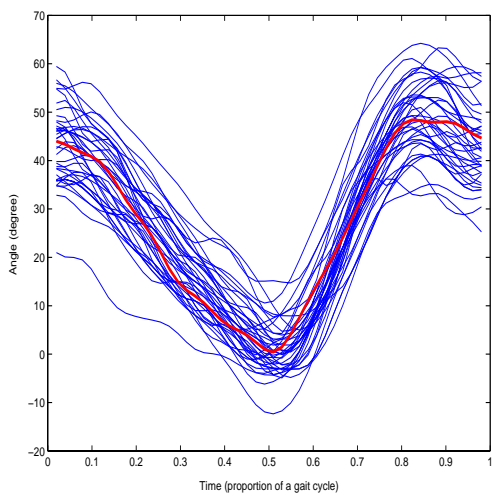

(a)

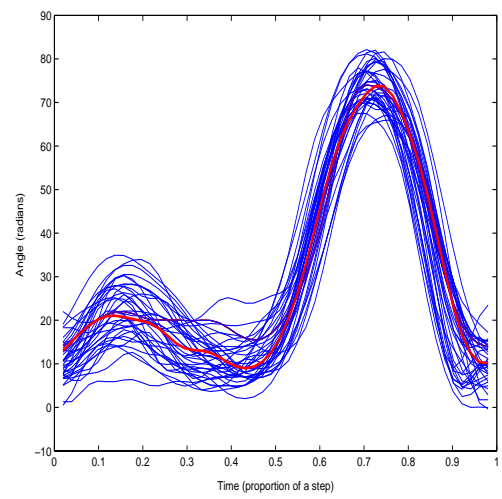

(b)

Figure 2: Angle in the sagittal plane formed by (a) the hip and (b) the knee, as 39 children go through a gait cycle. The deepest curves based on $S_{n, 2}$ within each sample are represented in red.

Definition 1. For functions $x_{1}, \ldots, x_{n}$, the band depth of any of these curves $x$ is

$$
S_{n, J}(x)=\sum_{j=2}^{J} S_{n}^{(j}(x), J \geq 2 .
$$

If $X_{1}, X_{2}, \ldots, X_{n}$ are independent copies of the stochastic process $X$ generating the observations $x_{1}, \ldots, x_{n}$, the corresponding population versions are $S^{(j}(x)=P\left\{G(x) \subset B\left(X_{i_{1}}, X_{i_{2}}, \ldots, X_{i_{j}}\right)\right\}$ and $S_{J}(x)=\sum_{j=2}^{J} S^{(j}(x)=\sum_{j=2}^{J} P\left\{G(x) \subset B\left(X_{i_{1}}, X_{i_{2}}, \ldots, X_{i_{j}}\right)\right\}$. A sample median function $\widehat{m}_{n, J}$ is a curve from the sample with highest depth value, $\widehat{m}_{n, J}=\underset{x \in\left\{x_{1}, \ldots, x_{n}\right\}}{\arg \max } S_{n, J}(x)$, and a population median is a function $m_{J}$ in $C(I)$ maximizing $S_{J}(\cdot)$. If they are not unique, the median will be the average of the curves maximizing depth.

The functions in Figure 2 provide the angle in the sagittal plane formed by the hip (left panel) and by the knee (right panel) as thirty nine children go through a gait cycle (see Ramsay and Silverman, 2005). The deepest curves (or median functions) for $S_{n, 2}$ appear in red (they are also the deepest ones for $2 \leq J \leq 7$ ). The notion of functional depth allows to order the data curves from the center-outward and, consequently, order based statistics can be defined. Thus, $L$-statistics will be generalized to the functional setting. After extensive simulation, this definition seems to be very stable with respect to the selection of the tuning parameter $J$. Figure 3 a is the multiple scatter plot of the ranks using $S_{J}$ for different values 


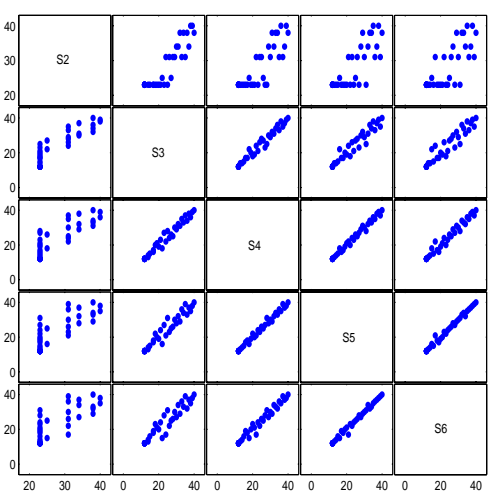

(a)

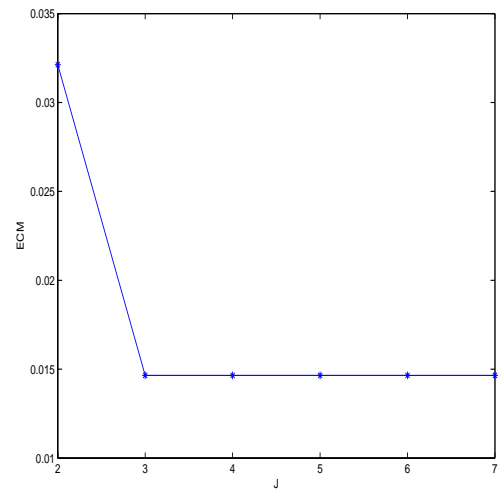

(b)

Figure 3: (a) Multiple scatterplot of the ranks of forty curves simulated from a gaussian process with $S_{J}, J=2, \ldots, 6$ and (b) mean integrated error for the deepest function.

of $J$. Forty sample paths in $C[0,1]$ have been simulated from a gaussian process with mean $m(t)=2 t$ and covariance function $\gamma(s, t)=\exp \left\{-|t-s|^{2}\right\}$. Their ranks were computed for $S_{2}, S_{3}, \ldots, S_{6}$. For $J$ larger than 3 , the points in the scatterplot fit the line $y=x$ and the ranks do not present dependence on $J$. To explore the mean integrated error, we have also simulated forty curves from a gaussian process in $C[0,1]$ with mean $f(t)=4 t$ and covariance function $\gamma(s, t)=\exp \left\{-|t-s|^{2}\right\}$. Figure 3b shows the mean integrated error $E I_{\widehat{m}}(J)=$ $\int_{0}^{1}\left(\widehat{m}_{n, J}(t)-f(t)\right)^{2} d t$ for different values of $J$, where $\widehat{m}_{n, J}(t)$ is the deepest observation for $S_{n, J}$. The mean integrated error is calculated as $E I_{\widehat{m}}(J)=\frac{1}{I} \sum_{k=1}^{I}\left[\widehat{m}_{n, J}(k / I)-f(k / I)\right]^{2}$ at $I=30$ points equally spaced in $[0,1]$. It is minimized for $J=3$ and remains constant for $J \geq 3$ : this indicates that the deepest point is the same for $J=3,4,5,6$ and 7 .

\section{FINITE-DIMENSIONAL VERSION}

The finite-dimensional version of the functional band depth provides also a depth for multivariate data. Parallel coordinates (see, e.g., Inselberg, 1981, 1985, Inselberg et al., 1987 and Wegman, 1990) provide a convenient way of visualizing a set of points in $\mathbb{R}^{d}$. The orthogonal axes in cartesian coordinates become parallel and equally spaced in parallel coordinates; thus, points with dimension larger than three can be easily represented. Observations in $\mathbb{R}^{d}$ can be seen as real functions defined on the set of indexes $\{1,2, \ldots, d\}$ and expressed as 
$x=(x(1), x(2), \ldots, x(d))$. Given points $x_{1}, x_{2}, \ldots, x_{n}$ in $\mathbb{R}^{d}$, the corresponding band in parallel coordinates $B\left(x_{1}, x_{2}, \ldots, x_{n}\right)=\left\{(k, y): k \in\{1,2, \ldots, d\}, \min _{i=1, \ldots, n} x_{i}(k) \leq y \leq \max _{i=1, \ldots, n} x_{i}(k)\right\}$ becomes a $d$-dimensional interval with sides parallel to the axes and delimited by the minimum and the maximum of the coordinates of $x_{1}, x_{2}, \ldots, x_{n}$,

$$
R\left(x_{1}, x_{2}, \ldots, x_{n}\right)=\left\{x \in \mathbb{R}^{d}: \min _{i=1, \ldots, n} x_{i}(k) \leq x(k) \leq \max _{i=1, \ldots, n} x_{i}(k)\right\} .
$$

Figures $4 \mathrm{a}$ and $4 \mathrm{~b}$ present the band delimited by three points in the plane in parallel and cartesian coordinates, respectively. For any point $x$ in $x_{1}, \ldots, x_{n}$,

$$
S_{n}^{(j}(x)=\left(\begin{array}{c}
n \\
j
\end{array}\right)^{-1} \sum_{1 \leq i_{1}<i_{2}<\ldots<i_{j} \leq n} I\left\{x \in R\left(x_{i_{1}}, x_{i_{2}}, \ldots, x_{i_{j}}\right)\right\}, j \geq 2,
$$

is the proportion of sets $R\left(x_{i_{1}}, x_{i_{2}}, \ldots, x_{i_{j}}\right)$ defined by $j$ different points $x_{i_{1}}, x_{i_{2}}, \ldots, x_{i_{j}}$ containing $x$. Hence, for points $x_{1}, \ldots, x_{n}$, the band depth of any of these points $x$ is

$$
S_{n, J}(x)=\sum_{j=2}^{J} S_{n}^{(j}(x), J \geq 2 .
$$

If $P$ is a probability distribution in $\mathbb{R}^{d}$ and $X_{1}, X_{2}, \ldots, X_{n}$ is a random sample from $P$, the band depth for any point $x$ in $\mathbb{R}^{d}$ with respect to $P$ is

$$
S_{J}(x)=S_{J}(x, P)=\sum_{j=2}^{J} P\left(x \in R\left(X_{i_{1}}, X_{i_{2}}, \ldots, X_{i_{j}}\right)\right), J \geq 2 .
$$

A sample median $\widehat{m}_{n, J}$ is a sample point with highest depth, $\widehat{m}_{n, J}=\underset{x \in\left\{x_{1}, \ldots, x_{n}\right\}}{\arg \max } S_{n, J}(x)$, and the population median $m_{J}$ is a point in $\mathbb{R}^{d}$ maximizing $S_{J}$. If there is more than one point with maximum depth, the median is defined as their mean. Figure 5a shows in red the four deepest points for $S_{3}$. The deepest observation is marked with a green circle. Also, rectangles determined by two and three points, respectively, can be seen in the same figure. The scatterplot in Figure 5b compares the ranks for thirty points in $\mathbb{R}^{2}$ using Mahalanobis, Tukey, Liu and $S_{3}$ depths. The deepest observation is usually the same for different depths and the orderings induced in the set of points are very similar. Contrary to most of previous definitions of depth, the band depth is not computationally intensive. This makes it convenient to analyze very high-dimensional data. 


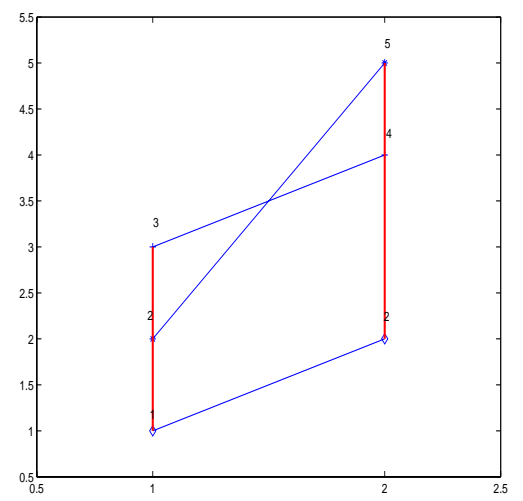

(a)

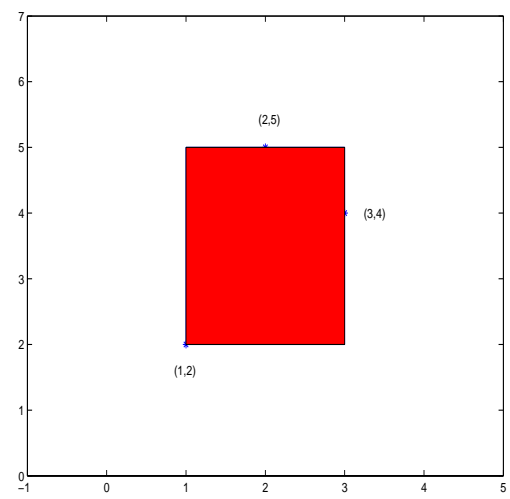

(b)

Figure 4: (a) Points $(1,2),(2,5)$ and $(3,4)$ and the corresponding band in parallel coordinates and (b) the same three points and band in cartesian coordinates.

\subsection{Band Depth Properties}

The deepest point for the band depth in one dimension is the usual univariate median; moreover, the order induced by the band depth in the real line does not depend on $J$. Liu (1990) established four natural properties a notion of depth should verify. Zuo and Serfling (2000) analyzed them in a very general framework. The band depth $S_{J}$ is a Type A depth function in their context (just write

$$
h\left(x, x_{1}, x_{2}, \ldots, x_{J}\right)=h_{2}\left(x, x_{1}, x_{2}\right)+h_{3}\left(x, x_{1}, x_{2}, x_{3}\right)+\ldots+h_{J}\left(x, x_{1}, x_{2}, \ldots, x_{J}\right),
$$

with $\left.h_{j}\left(x, x_{1}, x_{2}, \ldots, x_{j}\right)=I\left\{x \in R\left(x_{1}, x_{2}, \ldots, x_{j}\right)\right\}\right)$. Our first theorem gives the structural properties of the band depth: monotonicity, maximality at center, vanishing at infinity and continuity.

Theorem 1. Let $P$ be a probability distribution in $\mathbb{R}^{d}$. Then:

(i) If $P$ is absolutely continuous and its marginals $P_{i}, i=1,2, \ldots, d$ are symmetric with respect to the origin then $S_{J}(\alpha x)$ is a monotone nonincreasing function in $\alpha \geq 0$, for all $x \in \mathbb{R}^{d}$.

(ii) Under the conditions in (i), if the density $f$ is positive in a neighborhood of the center of symmetry $m$ then $S_{J}(\cdot)$ is uniquely maximized at $m$. 


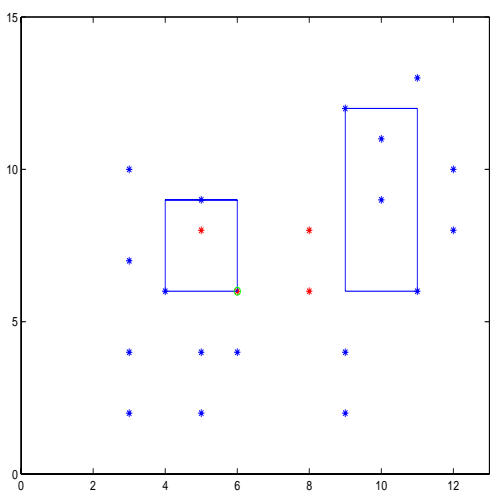

(a)

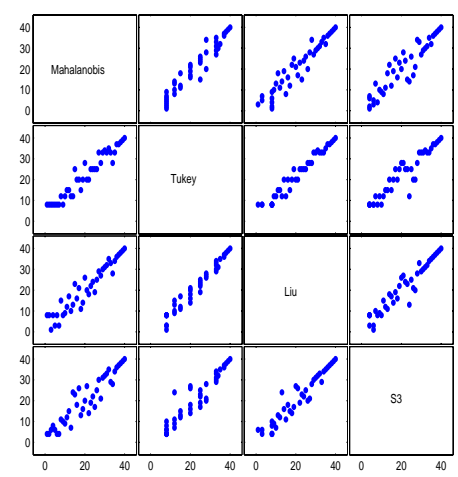

(b)

Figure 5: (a) Four deepest points from a sample in $\mathbb{R}^{2}$ are shown in red and the deepest one in green. Two rectangles determined by two and three points are also represented. (b) Multiple scatterplot diagram for the ranks of thirty points using Mahalanobis, Tukey, Liu and $S_{3}$ depths.

(iii) $\sup _{\|x\|_{\infty} \geq M} S_{J}(x) \longrightarrow 0$, as $M \rightarrow \infty$ and $\sup _{\|x\|_{\infty} \geq M} S_{n, J}(x) \stackrel{a . s}{\longrightarrow} 0$, as $M \rightarrow \infty$.

(iv) $S_{J}(\cdot)$ is upper- semicontinuous. If the marginal distributions of $P$ are absolutely continuous then $S_{J}(\cdot)$ is continuous.

The monotonicity in (i) is not verified if the underlying distribution is not absolutely continuous: let $d=1, P(X=0)=1 / 5, P(X= \pm 1)=1 / 5$, and $P(X= \pm 2)=1 / 5 ; X$ is symmetric with respect to $0, S_{2}(1 / 2 ; P)=12 / 25$ and $S_{2}(1 ; P)=15 / 25$.

Several interesting properties follow from the fact that the band depth is a $U$-statistic.

Proposition 1. $S_{J}(x)$ can be expressed as a $U$-statistic of order $J$.

The symmetry of $P$ is inherited by the sample distribution of the deepest point. Recall that a variable $X$ with distribution $P$ is antipodally symmetric with respect to $c$ if $(X-c)$ and $-(X-c)$ have the same distribution (see, e.g., Liu, Parelius and Singh, 1999).

Proposition 2. If $P$ is antipodally symmetric then the distribution of $\widehat{m}_{n, J}$ is also antipodally symmetric with respect to the population center of symmetry. 


\subsection{Asymptotic Results}

Theorem 2 provides the consistency properties of the band depth. Consider $S_{n}^{(j}(x)=$ $\left(\begin{array}{l}n \\ j\end{array}\right)^{-1} \sum_{1 \leq i_{i}<\ldots<i_{j} \leq n} I\left\{x \in R\left(X_{i_{1}}, \ldots, X_{i_{j}}\right)\right\}$, where $X_{i}$ are independent and identically distributed random variables taking values in $\mathbb{R}^{d}$ with distribution $P$ and $R\left(X_{1}, \ldots, X_{j}\right)$ is the closed interval in $\mathbb{R}^{d}$ defined by the points $X_{1}, \ldots, X_{j}$. Following Arcones and Giné (1993), $S_{n}^{(j}(x)$ is a $U$-process of order $j$ indexed by the class of functions $\mathcal{F}=\left\{R_{x}: x \in \mathbb{R}^{d}\right\}$, where $R_{x}$ $=\left\{I\left\{\left(x_{1}, \ldots, x_{j}\right): x \in R\left(x_{1}, \ldots, x_{j}\right)\right\}\right\}$. Then $S_{n}^{(j}(x)=U_{j}^{n}\left(R_{x}\right)$ and its population version is $S^{(j}(x)=P^{j}\left(x \in R\left(X_{1}, \ldots, X_{j}\right)\right)$.

Theorem 2. Let $P$ be a probability distribution in $\mathbb{R}^{d}$. Then:

(i) $\sup _{x \in \mathbb{R}^{d}}\left|S_{n, J}(x)-S_{J}(x)\right| \stackrel{\text { a.s. }}{\longrightarrow} 0$, as $n \rightarrow \infty$.

(ii) If $S_{J}(\cdot)$ is maximized at $m_{J}$ and $m_{n, J}$ is a sequence of random variables such that $S_{n, J}\left(m_{n, J}\right)=\sup _{x \in \mathbb{R}^{d}} S_{n, J}(x)$ then $m_{n, J} \stackrel{\text { a.s. }}{\longrightarrow} m_{J}$, as $n \rightarrow \infty$.

(iii) (Consistency of the sample deepest point) If the density $f$ is different from zero in a neighborhood of $m_{J}$ and $S_{J}(\cdot)$ is uniquely maximized at $m_{J}$ then $\widehat{m}_{n, J} \stackrel{a . s .}{\longrightarrow} m_{J}$, as $n \rightarrow \infty$.

A particular case of finite-dimensional observations are longitudinal data defined on discrete instants of time $t=1,2, \ldots, T$. All the definitions and properties presented in this section can be applied to this type of data.

\section{PROPERTIES OF THE FUNCTIONAL BAND DEPTH}

Next we analyze the band depth properties for functions. Let $X$ be a process in $C(I)$ with a tight distribution $P$, i.e., $P\left(\|X\|_{\infty} \geq M\right) \rightarrow 0$, as $M \rightarrow \infty$. The first result expresses the compatibility of the functional depth with the increasing finite-dimensional depth versions. Let $\mathbb{Q} \cap I=\left\{q_{1}, q_{2}, \ldots, q_{n}, \ldots\right\}$ be the sequence of rational numbers in $I$. Let $C_{m}$ be the set of vectors in $\mathbb{R}^{m}$ obtained evaluating a function $x$ at the first $m$ rational numbers of the interval $I . S_{J}$ denotes both the functional depth and the corresponding finite-dimensional version. The next result provides the convergence of $S_{J}\left(x_{m}\right)$ to $S_{J}(x)$ as $m$ tends to infinity, where $S_{J}\left(x_{m}\right)$ is the depth of $x_{m}$ in $C_{m}$. 
Proposition 3. If $x_{m}=\left(x\left(q_{1}\right), x\left(q_{2}\right), \ldots, x\left(q_{m}\right)\right)$ is the function $x$ evaluated at the first $m$ rational numbers in $I$ then $S_{J}\left(x_{m}\right)$ converges to $S_{J}(x)$, as $m$ tends to infinity.

The next Theorem gives the basic properties of the functional depth $S_{J}$.

Theorem 3.

(i) Let $T(x)=a x+b$, where $x, a$ and $b$ are continuous functions in $I$, with $a(t) \neq 0$ for each $t \in I$. Then $S_{J}(x, P)=S_{J}\left(a x+b, P_{a X+b}\right)$.

(ii) $\sup _{\|x\|_{\infty} \geq M} S_{J}(x) \longrightarrow 0$ and $\sup _{\|x\|_{\infty} \geq M} S_{n, J}(x) \stackrel{a . s}{\longrightarrow} 0$, as $M \rightarrow \infty$.

(iii) $S_{J}$ is an upper-semicontinuous function. Moreover, if the probability distribution $P$ on $C(I)$ has absolutely continuous marginal distributions, then the band depth $S_{J}$ is a continuous functional on $C(I)$.

Recall that a random variable $X$ on $C(I)$ is symmetric (with respect to the zero function) if $X$ and $-X$ have the same distribution.

Proposition 4. If the population random variable $X$ on $C(I)$ is symmetric then the distribution of $m_{n, J}$ is also symmetric.

The band depth is uniformly consistent on compact sets of functions.

Theorem 4. Let $P$ be a probability distribution on $C(I)$ with absolutely continuous marginal distributions. Then:

(i) $S_{n, J}(\cdot)$ is uniformly consistent on any equicontinuous set $E: \sup _{x \in E}\left|S_{n, J}(x)-S_{J}(x)\right| \stackrel{\text { a.s. }}{\rightarrow} 0$, as $n \rightarrow \infty$.

(ii) If $S_{J}(\cdot)$ is uniquely maximized at $m \in E$ and $m_{n}$ is a sequence of functions in $E$ with $S_{n, J}\left(m_{n}\right)=\sup _{x \in \mathbb{E}} S_{n, J}(x)$ then $m_{n} \stackrel{a . s .}{\longrightarrow} m$, as $n \rightarrow \infty$.

For instance, the set $\operatorname{Lip}_{\alpha, A}(I)=\left\{x: I \rightarrow \mathbb{R},\left|x\left(t_{1}\right)-x\left(t_{2}\right)\right| \leq A\left|t_{1}-t_{2}\right|^{\alpha}, t_{1}, t_{2} \in I\right\}$ is equicontinuous and verifies the condition in Theorem 4 ; hence, $S_{n, J}$ converges uniformly to $S_{J}$ over $\operatorname{Lip}_{\alpha, A}(I)$. The usual Lipschitzian functions are a particular case of $\operatorname{Lip}_{\alpha, A}(I)$ (with $\alpha=1$ ) and thus the band depth is uniformly consistent on the Lipschitz functions. 


\section{A GENERALIZED BAND DEPTH}

Instead of considering the indicator function, a more flexible definition can be introduced by measuring the set where the function is inside the corresponding band. For any of the functions $x$ in $x_{1}, \ldots, x_{n}$, let

$$
A_{j}(x) \equiv A\left(x ; x_{i_{1}}, x_{i_{2}}, \ldots, x_{i_{j}}\right) \equiv\left\{t \in I: \min _{r=i_{1}, \ldots, i_{j}} x_{r}(t) \leq x(t) \leq \max _{r=i_{1}, \ldots, i_{j}} x_{r}(t)\right\}, j \geq 2,
$$

be the set in the interval $I$ where the function $x$ is in the band determined by the observations $x_{i_{1}}, x_{i_{2}}, \ldots, x_{i_{j}}$. If $\lambda$ is the Lebesgue measure on $I, \lambda_{r}\left(A_{j}(x)\right)=\frac{\lambda\left(A_{j}(x)\right)}{\lambda(I)}$ gives the "proportion of time" that $x$ is in the band. Now,

$$
G S_{n}^{(j}(x)=\left(\begin{array}{c}
n \\
j
\end{array}\right)^{-1} \sum_{1 \leq i_{1}<i_{2}<\ldots<i_{j} \leq n} \lambda_{r}\left(A\left(x ; x_{i_{1}}, x_{i_{2}}, \ldots, x_{i_{j}}\right)\right), j \geq 2,
$$

is a generalized version of $S_{n}^{(j}(x)$ : if $x$ is always inside the band, the value $\lambda_{r}\left(A_{j}(x)\right)$ is one and this extends the previous definition.

Definition 2. For functions $x_{1}, \ldots, x_{n}$, the generalized band depth of any of these curves $x$ is

$$
G S_{n, J}(x)=\sum_{j=2}^{J} G S_{n}^{(j}(x), \quad J \geq 2 .
$$

If $X_{1}, X_{2}, \ldots, X_{n}$ are independent copies of the process $X$ giving the observations $x_{1}, \ldots, x_{n}$, the population version is $G S^{(j}(x)=E \lambda_{r}\left(A\left(x ; X_{i_{1}}, X_{i_{2}}, \ldots, X_{i_{j}}\right)\right), j \geq 2$, and $G S_{J}(x)=$ $\sum_{j=2}^{J} G S^{(j}(x), J \geq 2$. In the finite-dimensional case, $G S_{n}^{(j}(x)$ is the proportion of coordinates of $x$ inside the interval established by $j$ different points from the sample:

$G S_{n}^{(j}(x)=\left(\begin{array}{c}n \\ j\end{array}\right)^{-1} \sum_{1 \leq i_{1}<\ldots<i_{j} \leq n} \frac{1}{d} \sum_{k=1}^{d} I\left\{\min \left\{x_{i_{1}}(k), \ldots, x_{i_{j}}(k)\right\} \leq x(k) \leq \max \left\{x_{i_{1}}(k), \ldots, x_{i_{j}}(k)\right\}\right\}$.

In general, the order induced in a sample when $J$ increases is stable. To illustrate it we have simulated forty curves from a gaussian process defined on $[0,1]$ with zero mean and covariance function $\gamma(s, t)=\exp \left\{-|t-s|^{2}\right\}$. Figure 6 gives the multiple scatterplot of the curves ranks for the generalized band depth $G S_{J}$ with different values of $J$. The ranks present a good fit to the line $y=x$, and so they are essentially the same for different values 


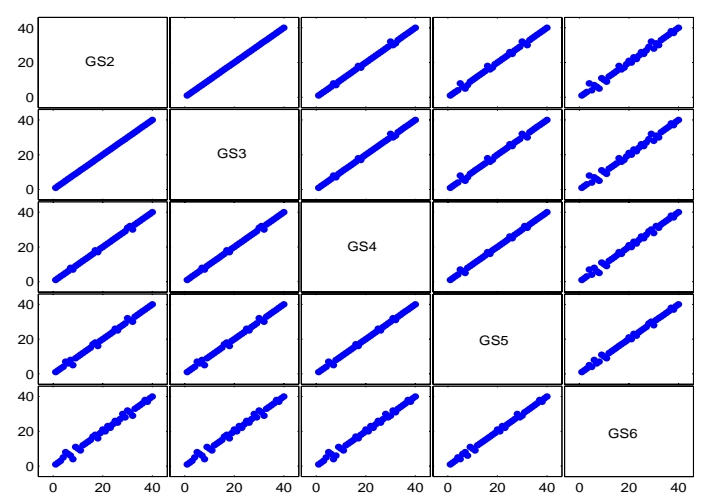

Figure 6: Multiple scatterplot diagram for ranks of forty curves simulated from a gaussian process based on $G S_{J}$ with $J=2,3,4,5,6$.

of $J$. Therefore, throughout the paper we will consider the generalized band depth with $J=2$ and it will be denoted as $G S$. For $J=2$,

$$
\begin{aligned}
G S_{n}(x) & =\frac{1}{d} \sum_{k=1}^{d}\left(\begin{array}{l}
n \\
2
\end{array}\right)^{-1} \sum_{1 \leq i_{1}<i_{2} \leq n} I\left\{\min \left\{x_{i_{1}}(k), x_{i_{2}}(k)\right\} \leq x(k) \leq \max \left\{x_{i_{1}}(k), x_{i_{2}}(k)\right\}\right\} \\
& =\frac{1}{d} \sum_{k=1}^{d} S D_{n} F_{k}(x(k)),
\end{aligned}
$$

where $S D_{F_{n, k}}(x(k))$ is the univariate simplicial depth of $x(k)$. Hence, $G S_{n}(x)$ is the average of the univariate simplicial depths for each coordinate $x(k)$. From the simplicial depth properties (see Liu, 1990) it is straightforward to check that the finite-dimensional version of the generalized band depth verifies all the properties established in Theorem 1, except (iii). The band depth is more dependent on the curves shape and more restrictive than the generalized version, which implies the presence of many ties. The generalized band depth relies more on the magnitude or size of the curves than on their shape. Another relevant difference between them is their behavior for curves leaving the center of the sample only for a short interval, i.e., remaining in the interior of the sample almost all the time, but taking extreme values in short subintervals: the generalized band depth can still be large for them but the band depth will always take small values on these curves. 

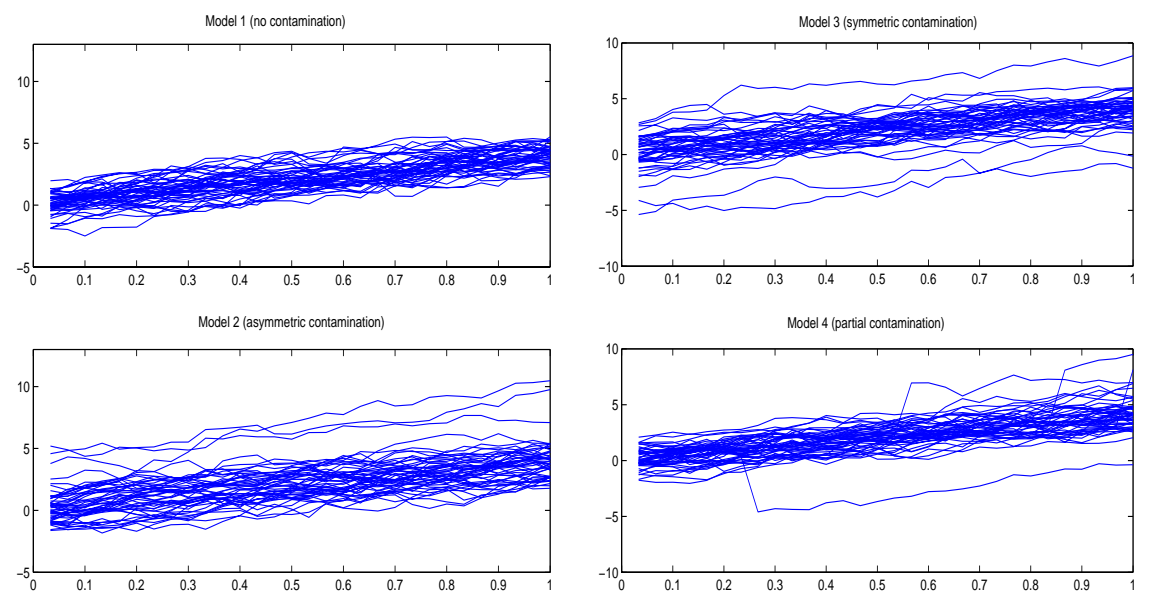

Figure 7: Curves generated from model 1 (without contamination), model 2 (asymmetric contamination), model 3 (symmetric contamination) and model 4 (partial contamination) with $M=5$ and $q=0.1$ in all cases.

\section{SIMULATION RESULTS}

We compare now the functional trimmed mean based on $S_{n, 3}$ and $G S_{n}$ with the mean in terms of robustness. We have generated curves from different models: a basic one without contamination and several models with different types of contaminations. They include those previously analyzed by Fraiman and Muniz (2001). Model 1 is the basic one, $X_{i}(t)=$ $g(t)+e_{i}(t), 1 \leq i \leq n$, where $e_{i}(t)$ is a stochastic gaussian process with zero mean and covariance function $\gamma(s, t)=\left(\frac{1}{2}\right)\left(\frac{1}{2}\right)^{5|t-s|}$ and $g(t)=4 t$, with $t \in[0,1]$. An asymmetric contamination appears in model $2, Y_{i}(t)=X_{i}(t)+c_{i} M, 1 \leq i \leq n$, where $c_{i}$ is 1 with probability $q$ and 0 with probability $1-q ; M$ is the contamination size constant. A symmetric contamination is included in model $3, Y_{i}(t)=X_{i}(t)+c_{i} \sigma_{i} M, 1 \leq i \leq n$, where $c_{i}$ and $M$ are defined in model 2 and $\sigma_{i}$ is a sequence of random variables independent of $c_{i}$ taking values 1 and -1 with probability $1 / 2$. Model 4 is partially contaminated, $Y_{i}(t)=X_{i}(t)+c_{i} \sigma_{i} M$, if $t \geq$ $T_{i}, 1 \leq i \leq n$, and $Y_{i}(t)=X_{i}(t)$, if $t<T_{i}$, where $T_{i}$ is a random number generated from

a uniform distribution on $[0,1]$. Curves simulated from these four models can be seen in Figure 7.

Model 5 is contaminated by peaks, $Y_{i}(t)=X_{i}(t)+c_{i} \sigma_{i} M$, if $T_{i} \leq t \leq T_{i}+l, 1 \leq i \leq$ $n$, and $Y_{i}(t)=X_{i}(t)$, if $t \notin\left[T_{i}, T_{i}+l\right]$, where $l=2 / 30$ and $T_{i}$ is a random number from 


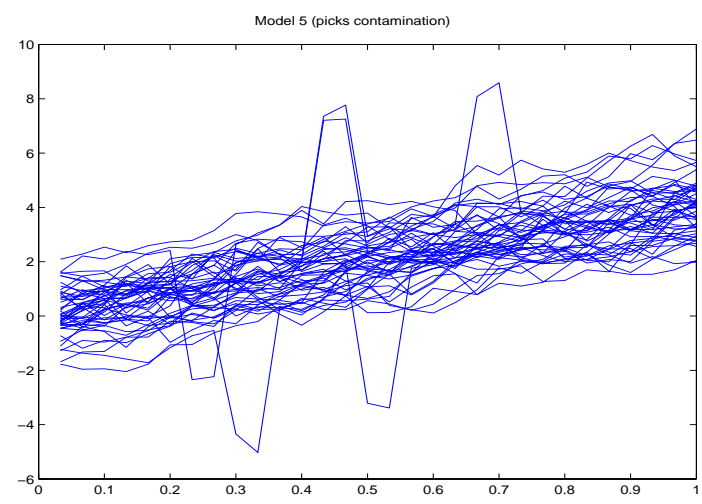

Figure 8: Fifty curves from model $5(M=5$ and $q=0.1)$.

a uniform distribution in $[0,1-l]$. The contamination only occurs for a short subinterval of length $l$. Figure 8 shows an example of model 5 curves. Our goal is to analyze the robustness of statistics based on functional depth. We compare the mean and the $\alpha$-trimmed mean, given by $\widehat{\mu}_{n}(t)=\frac{\sum_{i=1}^{n} Y_{i}(t)}{n}$ and $\widehat{m}_{n}^{\alpha}(t)=\frac{\sum_{i=1}^{n-[n \alpha]} Y_{(i)}(t)}{n-[n \alpha]}$, where $Y_{(1)}, Y_{(2)}, \ldots Y_{(n)}$ is the sample ordered from the deepest to the least deep curve and $[n \alpha]$ is the integer part of $n \alpha$. For each model, we have considered $R$ replications, generating $n$ curves in each replication and calculating the integrated error evaluated at $T=30$ equally spaced points in $[0,1]$. The integrated errors for each replication $j$ are $E I_{\mu}(j)=\frac{1}{T} \sum_{k=1}^{T}\left[\widehat{\mu}_{n}(k / T)-g(k / T)\right]^{2}$ and $E I_{S}^{\alpha}(j)=$ $\frac{1}{T} \sum_{k=1}^{T}\left[\widehat{m}_{n}^{\alpha}(k / T)-g(k / T)\right]^{2}$, respectively. Table 1 contains the mean integrated error for each model considering $R=500$ replications with $n=50$ curves, contamination probability $q=0.1$, two contamination constants, $M=5, M=25$ and $\alpha=0.2$. In the model without contamination the mean behaves always better than the trimmed mean. For models 2, 3 and 4 the best mean integrated error corresponds to $G S$ (and with $S_{3}$ the results improve over the mean); however, for model 5 with $M=25$, the mean integrated error is minimized for $S_{3}$. The reason is that contamination in model 5 appears in a small domain interval and the generalized depth is not very robust with respect to this type of contamination; a curve with contaminated values in a short interval of the domain can still be deep using the generalized band depth whereas it will not be deep based on the band depth. 


\begin{tabular}{|c|c|c|c|c|c|c|}
\hline Const. & Estimator & Model 1 & Model 2 & Model 3 & Model 4 & Model 5 \\
\hline \multirow{2}{*}{$M=5$} & \multirow{2}{*}{ Mean } & 0.0094 & 0.3106 & 0.0578 & 0.0322 & 0.0153 \\
\hline & & $(0.0067)$ & $(0.2564)$ & $(0.0656)$ & $(0.0326)$ & $(0.0086)$ \\
\hline & \multirow{2}{*}{$S_{3}$} & 0.0126 & 0.2519 & 0.0267 & 0.0314 & 0.0179 \\
\hline & & $(0.0096)$ & $(0.2584)$ & $(0.0290)$ & $(0.0313)$ & $(0.0113)$ \\
\hline & \multirow{2}{*}{$G S$} & 0.0139 & 0.0228 & 0.0168 & 0.0184 & 0.0192 \\
\hline & & $(0.0104)$ & $(0.0359)$ & $(0.0112)$ & $(0.0142)$ & $(0.0113)$ \\
\hline \multirow{2}{*}{$\mathrm{M}=25$} & \multirow{2}{*}{ Mean } & 0.0099 & 2.1158 & 0.7634 & 0.3296 & 0.1442 \\
\hline & & $(0.0069)$ & $(2.1485)$ & $(1.0839)$ & $(0.4674)$ & $(0.0769)$ \\
\hline & \multirow{2}{*}{$S_{3}$} & 0.0128 & 1.1345 & 0.2907 & 0.2558 & 0.0876 \\
\hline & & $(0.0094)$ & $(1.8690)$ & $(0.7061)$ & $(0.3944)$ & $(0.0812)$ \\
\hline & \multirow{2}{*}{$G S$} & 0.0129 & 0.0781 & 0.0148 & 0.0951 & 1.3762 \\
\hline & & $(0.0110)$ & $(0.3481)$ & $(0.0109)$ & $(0.1587)$ & $(0.0662)$ \\
\hline
\end{tabular}

Table 1: Means and standard deviations of integrated errors with $R=500$ replications, $q=0.1$ and $\alpha=0.2$. 


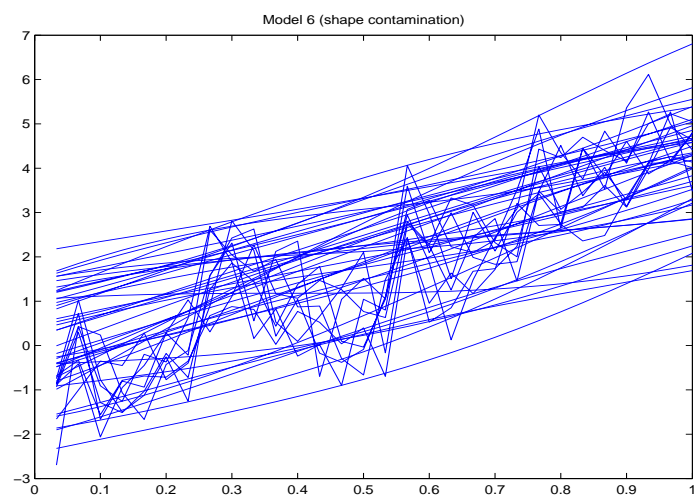

Figure 9: Curves from model 6 with $k_{2}=c_{2}=1, \mu_{2}=0.2$ and contamination probability $q=0.1$.

Besides magnitude contamination, we have also considered shape contamination. There is no general definition of outlier for functional observations. A curve could be outlier for different reasons: it can be very distant from the mean (magnitude outlier) or have a pattern different from the other curves, being, e.g., decreasing when the remaining ones are increasing or very irregular in a set of smooth curves (shape outlier). To generate shape outliers, we use the covariance $\gamma(s, t)=k \exp \left\{-c|t-s|^{\mu}\right\}$, with $s, t \in[0,1]$, and $k, c$, $\mu>0$ (see Wood and Chan, 1994). Different values of $k, c$ and $\mu$ change the shape of the generated functions. For example, increasing $\mu$ and $k$, the curves are smoother; however, increasing $c$ the curves are more irregular. Model 6 is a mixture of $X_{i}(t)=g(t)+e_{1 i}(t)$, $1 \leq i \leq n$, with $g(t)=4 t$ and $e_{i 1}(t)$ a gaussian stochastic process with zero mean and covariance function $\gamma_{1}(s, t)=\exp \left\{-|t-s|^{2}\right\}$ and $Y_{i}(t)=g(t)+e_{2 i}(t), 1 \leq i \leq n$, with $e_{i 2}(t)$ a gaussian process with zero mean and covariance function with values $k_{2}, c_{2}$ and $\mu_{2}$ chosen to generate more irregular curves (for example, $\mu_{2}<2$ or $c_{2}>1$ ). The contaminated model 6 is $Z_{i}(t)=(1-\varepsilon) X_{i}(t)+\varepsilon Y_{i}(t), 1 \leq i \leq n$, where $\varepsilon$ is a Bernoulli variable $B e(q)$ and $q$ is a small contamination probability; thus, we contaminate a sample of smooth curves from $X_{i}(t)$ with curves from $Y_{i}(t)$ having different covariance function and providing more irregular curves. Figure 9 shows curves simulated from model 6: the contaminated curves behave more irregularly than the remaining functions but they are not far from them in terms of distance. Table 2 contains the simulation results for model 6 with $R=500, n=50$ 


\begin{tabular}{|c|c|c|c|c|}
\hline & Estimation & $\begin{array}{c}\mu_{2}=0.2 \\
k_{2}=1\end{array}$ & $\begin{array}{c}\mu_{2}=0.1 \\
k_{2}=1\end{array}$ & $\begin{array}{c}\mu_{2}=0.1 \\
k_{2}=2\end{array}$ \\
\hline \multirow[t]{3}{*}{$q=0.15$} & Mean & $\begin{array}{c}0.0455 \\
(0.0487)\end{array}$ & $\begin{array}{c}0.0483 \\
(0.0622)\end{array}$ & $\begin{array}{c}0.0468 \\
(0.0418)\end{array}$ \\
\hline & $S_{3}$ & $\begin{array}{c}0.0321 \\
(0.0455)\end{array}$ & $\begin{array}{c}0.0295 \\
(0.0351)\end{array}$ & $\begin{array}{r}0.0250 \\
(0.0233)\end{array}$ \\
\hline & $G S$ & $\begin{array}{c}0.0542 \\
(0.0633)\end{array}$ & $\begin{array}{c}0.0488 \\
(0.0447)\end{array}$ & $\begin{array}{c}0.0364 \\
(0.0339)\end{array}$ \\
\hline \multirow[t]{3}{*}{$q=0.1$} & Mean & $\begin{array}{c}0.0318 \\
(0.0334)\end{array}$ & $\begin{array}{c}0.0360 \\
(0.0320)\end{array}$ & $\begin{array}{c}0.0355 \\
(0.0297)\end{array}$ \\
\hline & $S_{3}$ & $\begin{array}{c}0.0293 \\
(0.0344)\end{array}$ & $\begin{array}{c}0.0282 \\
(0.0313)\end{array}$ & $\begin{array}{r}0.0272 \\
(0.0299)\end{array}$ \\
\hline & $G S$ & $\begin{array}{c}0.0345 \\
(0.0508)\end{array}$ & $\begin{array}{c}0.0414 \\
(0.0369)\end{array}$ & $\begin{array}{c}0.0380 \\
(0.0381)\end{array}$ \\
\hline
\end{tabular}

Table 2: Simulation results with $R=500$ replications, $n=50$ curves and $\alpha=0.2$.

and $\alpha=0.2$. We have considered two contamination probabilities ( $q=0.15$ and $q=0.1$ ) and different values of $\mu_{2}, k_{2}$ to modify the covariance of $Y(t)\left(c_{2}=1\right.$ in all cases). The minimum mean integrated error corresponds always to the trimmed mean based on the band depth $S_{3}$. This is due to the contamination type, shape more than magnitude; thus, the band depth performs well in terms of robustness with respect to this form of outliers. However, the generalized band depth is less robust against shape contamination, since most of the contaminated curves values can still be very central in the sample although the curve behavior is different from the remaining functions.

\section{REAL DATA EXAMPLES}

The first real data set describes daily temperature in different Canadian weather stations (Ramsay and Silverman, 2005). The original data was smoothed using a Fourier basis with sixty five elements. Figure 10a shows the temperature curves; the five deepest functions for the band depth are in red. Figure 10b presents the five deepest curves using the generalized 


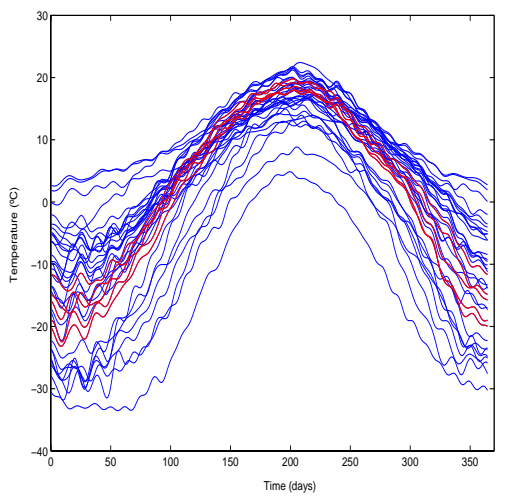

(a)

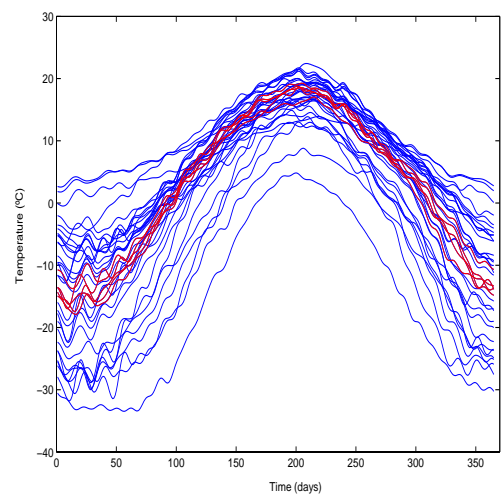

(b)

Figure 10: Daily temperature in 35 Canadian weather stations for one year. The five deepest curves (in red) based on (a) $S_{n, 3}$ and (b) GS.

band depth. The second example contains the price curves for the 35 firms in the Spanish IBEX 35 index. The functions include fifty nine daily measurements starting at June 22, 2002. Figure 11a gives in red the $30 \%$ deepest elements for $S_{3}$. The mean curve is represented with blue asterisks and the trimmed mean with $\alpha=0.3$ appears in green asterisks. The mean curve is more sensitive to the extreme functions than the trimmed one. In Figure 11b the $25 \%$ most extreme (least deep) curves from the sample are in red.

\section{A RANK TEST FOR FUNCTIONS}

The depth definition for curves allows us to extend the rank test to functional data. Liu and Singh (1993) generalized to multivariate data the univariate Wilcoxon rank test through the order induced by a multivariate depth. Brown and Hettmansperger (1989) and Hettmansperger and Oja (1994) have also proposed different rank tests for multivariate observations. Following Liu and Singh (1993), let

$$
R\left(P_{n}, x_{i}\right)=R\left(x_{i}\right)=\text { proportion of } x_{j} \text { 's from the sample with } S\left(x_{j}\right) \leq S\left(x_{i}\right)
$$

It takes values between 0 and 1 . We rank the observations $x_{i}$ according to the increasing values of $R$, assigning them an integer from 1 to $n$. If there are curves with the same value of $R, R\left(x_{i_{1}}\right)=R\left(x_{i_{2}}\right)=\ldots=R\left(x_{i_{j}}\right)$, with $i_{1}<i_{2}<\ldots<i_{j}$, we consider the rank of $x_{i_{k+1}}$ as the rank of $x_{i_{k}}$ plus one. We propose a test based on these ranks to decide if 


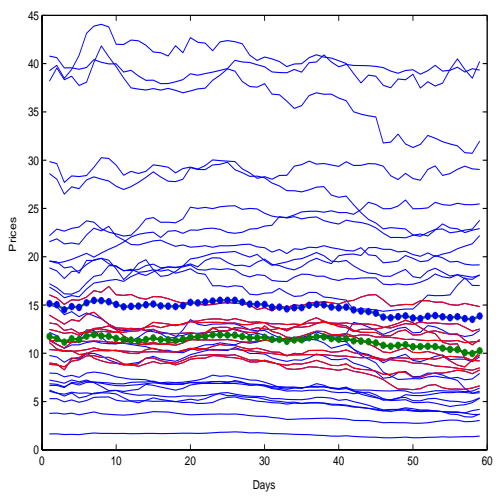

(a)

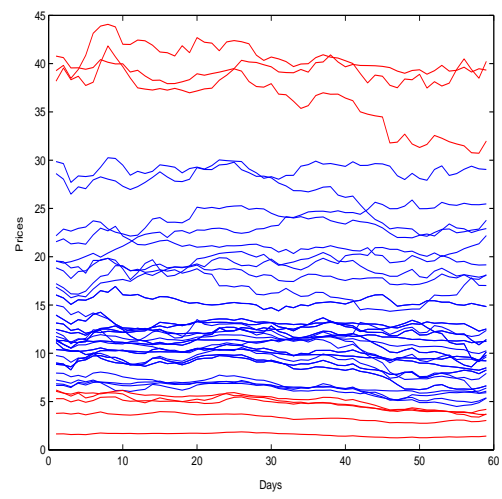

(b)

Figure 11: Prices included in the IBEX 35 index. (a) The $30 \%$ deepest curves are in red, and mean and median are marked with blue and green asterisks, respectively. (b) The $25 \%$ most extreme curves appear in blue.

two groups of curves come from the same population. Let $x_{1}, \ldots, x_{n}$ be a sample of curves from population $P_{1}$ and let $y_{1}, \ldots, y_{m}$ be a sample of curves from population $P_{2}$. Assume that there is a third reference sample $Z=\left\{z_{1}, z_{2}, \ldots, z_{n_{0}}\right\}$ from one of the two populations, for example $P_{1}$, with $n_{0}$ greater than $n$ and $m$. Let $P_{n_{0}}$ be the corresponding empirical distribution. Calculate $R\left(P_{n_{0}}, x_{i}\right)=$ proportion of $z_{j}$ 's with $S\left(z_{j}, P_{n_{0}}\right) \leq S\left(x_{i}, P_{n_{0}},\right)$ and $R\left(P_{n_{0}}, y_{i}\right)=$ proportion of $z_{j}$ 's with $S\left(z_{j}, P_{n_{0}}\right) \leq S\left(y_{i}, P_{n_{0}}\right)$, that express the position of each $x_{i}$ and $y_{i}$ with respect to $Z$. Order these values, $R\left(P_{n_{0}}, X_{i}\right)$ and $R\left(P_{n_{0}}, Y_{i}\right)$, from smallest to highest giving them a rank from 1 to $n+m$. If there are ties, we apply the previous criterion. The proposed statistic to test $H_{0}: P_{1}=P_{2}$ is $W=\sum_{j=1}^{m} \operatorname{ranks} R\left(P_{n_{0}}, y_{j}\right)$. The ranks of $R\left(P_{n_{0}}, y_{j}\right)$ behave under $H_{0}$ as $m$ numbers randomly chosen from $\{1,2, \ldots, n+m\}$. Hence, the distribution of $W$ is the distribution of $\rho_{1}+\ldots+\rho_{m}$, where $\rho_{1}, \ldots, \rho_{m}$ is a sample without replacement of $\{1,2, \ldots, n+m\}$ (see Liu and Singh, 1993). The null hypothesis is rejected when $W$ is small, because this indicates that $R\left(P_{n_{0}}, y_{j}\right)$ take on average lower values than $R\left(P_{n_{0}}, x_{i}\right)$, implying that the observations $y_{j}$ are less deep with respect to $P_{n_{0}}$ than $x_{i}$. The alternative hypothesis is that on average more than $50 \%$ of population $P_{1}$ is inner or more central than any observation from $P_{2}$, indicating that the distributions are not the same.

We have applied this test to real data representing the relative diameter versus the 
relative height of two groups of trees (Laricio and Radiata). Due to technical restrictions in the measurements, the relative diameter is defined as the ratio between its value at the corresponding height and the diameter at a fix height (1.3 centimeters). Relative height is height over the total height of the tree. Figure 12 shows the curves corresponding to seventy Laricio trees (left panel) and one hundred and forty Radiata trees (right panel). Since the number of observations per tree is very irregular (from 3 to 25), the data has been smoothed using a spline basis. To apply the rank test, we have considered seventy functions randomly chosen from the Radiata trees group as the reference group to compute the ranks of the remaining curves. The obtained $p$-values, using both the band depth and the generalized band depth are very close to zero. Therefore, we conclude that there exists significative differences between both groups. The second real data set includes the growth curves for boys and girls (see Ramsay and Silverman, 2005). We have applied the rank test to decide if there are no differences between both groups curves. We consider thirty two curves randomly chosen from the group of girls as the reference group. The remaining twenty two curves constitute the test group together with the thirty growth curves for boys. The obtained $p$-value with $S_{3}$ is 0.00011 ; hence, we reject the null hypothesis, concluding that there exist significative differences between the growth curves for boys and girls. This difference could be caused by a change either in mean or in dispersion. Graphically, the groups do not seem to be similar (see Figure 13). The shape is different and the heights of boys achieve higher values at the end. The rank test detects these differences. However, if we apply the rank test using the generalized band depth $(G S)$ or Fraiman and Muniz's depth (FM) instead of $S_{3}$, we obtain $p$-values of 0.1199 and 0.1636 respectively, concluding in this case that there is no evidence for rejecting the null hypothesis. The reason is that $G S$ and FM consider only the magnitudes, ignoring the curves shape. Note that the average values of boys and girls heights only differ in the final years (17 to 18 years) and $F M$ and $G S$ do not detect differences between both groups because they occur over a short interval. Since the essential difference between both groups is shape, we have applied the rank test to the curves derivatives (growth speed) (see Figure 14). The rank test $p$-values using $S_{3}$, $G S$ and $F M$ are very close to zero $\left(1.6 * 10^{-4}, 4.54 * 10^{-7}\right.$ and $6.01 * 10^{-7}$, respectively) and the null hypothesis is rejected. The instant of growth maximum velocity is different for boys 

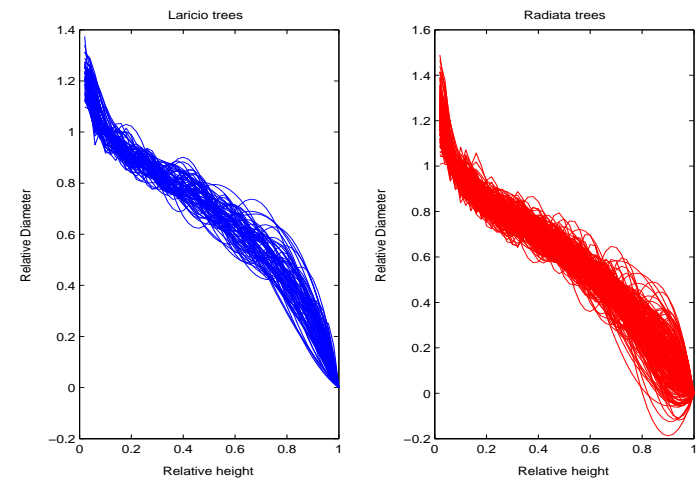

Figure 12: Relative diameter versus relative height for Laricio trees represented in blue (left panel) and for Radiata trees represented in red (right panel).
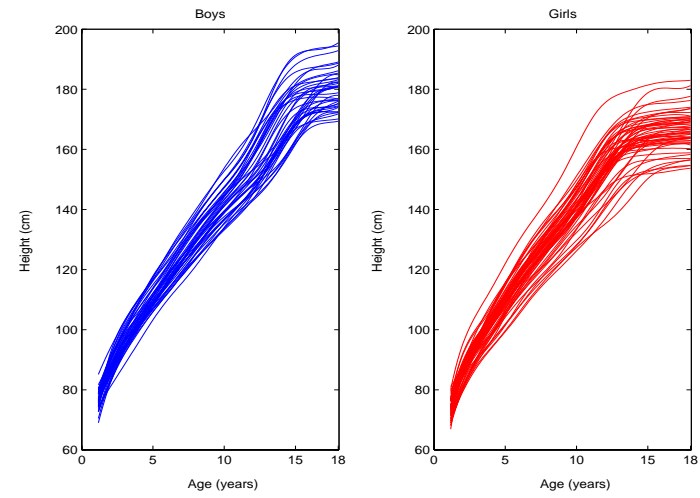

Figure 13: Growth curves for a sample of thirty boys (left panel) and fifty four girls (right panel).

and girls (see Figure 14). Girls reach maximum speed at an earlier age than boys. The band depth rank test detects these shape differences for boys and girls both in the original sample of growth curves and in the derivatives set.

\section{CONCLUSIONS}

We have introduced a band depth for functional data based on the graphic representation of the curves. It provides a criterion to order a sample of functions from center-outward and robust statistics for functional observations, such as the median and trimmed mean, can be constructed. Its finite-dimensional version provides a new multivariate depth. It is very 


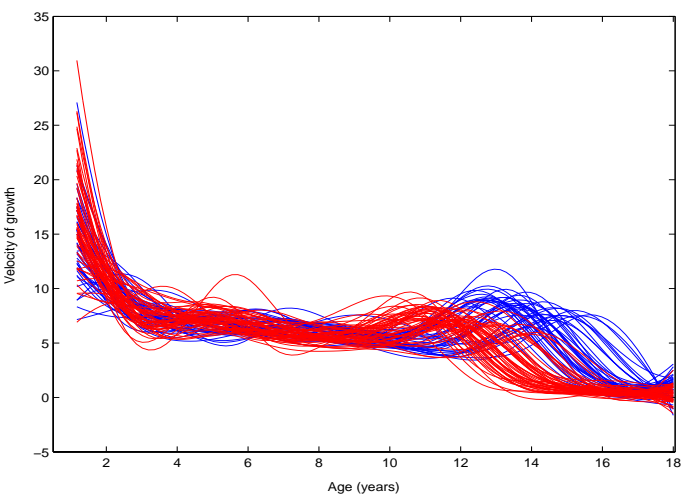

Figure 14: First derivative of the growth curves for boys (in blue) and girls (in red).

convenient to deal with high-dimensional data since it is computationally very fast, avoiding the main drawback of other finite-dimensional depth definitions. This new notion verifies natural depth properties. We have also established the uniform consistency of the sample depth in the finite and functional case. We have illustrated the robustness of this new depth with a simulation study and several real examples. As an application, a rank test for functional data is introduced and applied to decide whether two groups of curves come from the same population.

\section{APPENDIX: PROOFS}

Proof of Theorem 1: (i) The proof follows along the lines of Liu (1990). We check it for $d=2$ and analyze the events contributing to the difference $S^{(j}(x)-S^{(j}(\alpha x)$, with $\alpha \geq 1$. Consider the sets defined by the arrow from $x$ to $\alpha x$ entering or leaving $R\left(X_{1, \ldots,} X_{j}\right)$ with sides parallel to the axes. Given two points $a$ and $b$, the line containing the segment $\overline{a b}$ divides the plane into two half planes. If the origin does not belong to this line, the half plane including the origin is called the inner side $I(a, b)$. Let $C=\{(a, b): \overline{a b} \cap \overline{x, \alpha x} \neq \emptyset\}$; hence, $C$ is the set of all segments that intersect the segment $\overline{x, \alpha x}$. Define the sets $r_{1}=$

$$
\begin{aligned}
& \left(\min _{i=1, \ldots, j}\left\{X_{i}(1)\right\}, \min _{i=1, \ldots, j}\left\{X_{i}(2)\right\}\right), r_{2}=\left(\max _{i=1, \ldots, j}\left\{X_{i}(1)\right\}, \min _{i=1, \ldots, j}\left\{X_{i}(2)\right\}\right) \\
& r_{3}=\left(\max _{i=1, \ldots, j}\left\{X_{i}(1)\right\}, \max _{i=1, \ldots, j}\left\{X_{i}(2)\right\}\right), r_{4}=\left(\min _{i=1, \ldots, j}\left\{X_{i}(1)\right\}, \max _{i=1, \ldots, j}\left\{X_{i}(2)\right\}\right) .
\end{aligned}
$$

We have that $x \in R\left(X_{1}, \ldots, X_{j}\right)$ is equivalent to express that $x$ belongs to the rectangle 
determined by the random points $r_{1}, r_{2}, r_{3}, r_{4}$. Consider the disjoint sets

$$
\begin{array}{ll}
A_{i n}^{r_{1} r_{2}}=\left\{\left(X_{1}, \ldots, X_{j}\right)\right. \text { s.t. } & \left.\left(r_{1}, r_{2}\right) \in C \text { and } r_{3} \notin I\left(r_{1}, r_{2}\right)\right\} \\
A_{i n}^{r_{2} r_{3}}=\left\{\left(X_{1}, \ldots, X_{j}\right)\right. \text { s.t. } & \left.\left(r_{2}, r_{3}\right) \in C \text { and } r_{4} \notin I\left(r_{2}, r_{3}\right)\right\} \\
A_{i n}^{r_{3} r_{4}}=\left\{\left(X_{1}, \ldots, X_{j}\right)\right. \text { s.t. } & \left.\left(r_{3}, r_{4}\right) \in C \text { and } r_{1} \notin I\left(r_{3}, r_{4}\right)\right\} \\
A_{i n}^{r_{4} r_{1}}=\left\{\left(X_{1}, \ldots, X_{j}\right)\right. \text { s.t. } & \left.\left(r_{4}, r_{1}\right) \in C \text { and } r_{2} \notin I\left(r_{4}, r_{1}\right)\right\} .
\end{array}
$$

Denote by $A_{\text {in }}$ the events where the vector $\overline{x, \alpha x}$ enters a random rectangle with vertexes $\left(r_{1}, r_{2}, r_{3}, r_{4}\right)$. Therefore, $A_{i n}=A_{i n}^{r_{1} r_{2}} \cup A_{i n}^{r_{2} r_{3}} \cup A_{i n}^{r_{3} r_{4}} \cup A_{i n}^{r_{4} r_{1}}$. The events $A_{\text {out }}$ are those where the arrow $\overline{x, \alpha x}$ leaves a random rectangle, and can be decomposed as a union of disjoint events in a similar way as $A_{i n}$. Let $B_{\alpha}=\left\{\alpha x \in R\left(X_{1}, \ldots, X_{j}\right)\right\}$. Since $P\left(B_{1} \backslash B_{\alpha}\right)=$ $P\left(B_{1}\right)-P\left(B_{1} \cap B_{\alpha}\right), S^{(j}(x)-S^{(j}(\alpha x)=P\left(B_{1} \backslash B_{\alpha}\right)-P\left(B_{\alpha} \backslash B_{1}\right)$. Now, $B_{1} \backslash B_{\alpha}=$ $A_{\text {out }} \backslash A_{\text {in }}$ and $B_{\alpha} \backslash B_{1}=A_{\text {in }} \backslash A_{\text {out }}, S^{(j}(x)-S^{(j}(\alpha x)=P\left(A_{\text {out }} \backslash A_{\text {in }}\right)-P\left(A_{\text {in }} \backslash A_{\text {out }}\right)=$ $P\left(A_{\text {out }}\right)-P\left(A_{\text {out }} \cap A_{\text {in }}\right)-\left[P\left(A_{\text {in }}\right)-P\left(A_{\text {in }} \cap A_{\text {out }}\right)\right]=P\left(A_{\text {out }}\right)-P\left(A_{\text {in }}\right)$, and it is sufficient to prove that $P\left(A_{\text {out }}^{r_{i} r_{j}}\right)-P\left(A_{i n}^{r_{i} r_{j}}\right)$ is positive for each $i, j$ in (6). We present the proof only for one of them. For example, $P\left(A_{\text {out }}^{r_{1} r_{2}}\right)-P\left(A_{\text {in }}^{r_{1} r_{2}}\right)=$

$$
=\int_{\left\{\left(x_{1}, \ldots, x_{j}\right):\left(r_{1}, r_{2}\right) \in C\right\}}\left\{P\left(r_{3} \in I\left(r_{1}, r_{2}\right)\right)-P\left(r_{3} \notin I\left(r_{1}, r_{2}\right)\right)\right\} d F\left(x_{1}\right) \ldots d F\left(x_{j}\right)
$$

and it will be positive when $P\left(r_{3} \in I\left(r_{1}, r_{2}\right)\right) \geq 1 / 2$, since this implies that

$$
\left\{P\left(r_{3} \in I\left(r_{1}, r_{2}\right)\right)-P\left(r_{3} \notin I\left(r_{1}, r_{2}\right)\right)\right\} \geq 0
$$

If the second coordinate of $r_{1}$ is positive, we will denote this event as $r_{1} u p$, and if it is negative, $r_{1}$ down. So,

$$
\begin{gathered}
P\left(r_{3} \in I\left(r_{1}, r_{2}\right)\right)=P\left(r_{3} \in I\left(r_{1}, r_{2}\right) \mid r_{1} \text { up }\right) \times P\left(r_{1} \text { up }\right)+ \\
+P\left(r_{3} \in I\left(r_{1}, r_{2}\right) \mid r_{1} \text { down }\right) \times P\left(r_{1} \text { down }\right)=0+P\left(r_{1} \text { down }\right) .
\end{gathered}
$$

Now, by the definition of $r_{1}$, and due to the symmetry with respect to the origin,

$$
\begin{aligned}
P\left(r_{1} \text { down }\right) & \left.\left.=P\left(\min \left\{X_{1}(2), \ldots, X_{j}(2)\right\}<0\right)\right)=1-P\left(\min \left\{X_{1}(2), \ldots, X_{j}(2)\right\}>0\right)\right)= \\
& =1-\left[P\left(X_{1}(2)>0\right) \times \ldots \times P\left(X_{j}(2)>0\right)\right]=1-\left(\frac{1}{2}\right)^{j}>\frac{1}{2} .
\end{aligned}
$$

Then, for $\alpha \geq 1, S^{j)}(x)-S^{j)}(\alpha x) \geq 0$; thus, $S^{j)}(\alpha x)$ is non increasing in $\alpha$, for $\alpha \geq 0$. Hence, $S_{J}(\alpha x)$ is also nonincreasing in $\alpha$, for $\alpha \geq 0$. 
(iii) We establish first the inclusion

$$
\left\{\left(X_{1}, X_{2}, \ldots, X_{j}\right): x \in R\left(X_{1}, X_{2}, \ldots, X_{j}\right)\right\} \subset \bigcup_{r=1}^{j}\left\{\left(X_{1}, X_{2}, \ldots, X_{j}\right):\left\|X_{r}\right\|_{\infty} \geq\|x\|_{\infty}\right\}
$$

by contradiction. If $x \in R\left(X_{1}, X_{2}, \ldots, X_{j}\right)$ then, for each $k \in\{1, \ldots, d\}$,

$$
\min _{r=1, \ldots, j}\left\{X_{r}(k)\right\} \leq x(k) \leq \max _{r=1, \ldots, j}\left\{X_{r}(k)\right\} .
$$

Assume that $\left\|X_{r}\right\|_{\infty}<\|x\|_{\infty}$ for each $r=1, \ldots, j$; this implies that, for each $r$, we have

$$
\max _{k \in\{1, \ldots, d\}}\left|X_{r}(k)\right|<\max _{k \in\{1, \ldots, d\}}|x(k)|
$$

Let $k^{*}$ be the point where the maximum of $x(k)$ is achieved. Then, for all $r=1, \ldots, j$, $\left|X_{r}\left(k^{*}\right)\right|<\left|x\left(k^{*}\right)\right|$, and this contradicts (8). Therefore,

$$
\begin{aligned}
\sup _{\|x\|_{\infty} \geq M} S_{J}(x) & \leq \sum_{j=2}^{J} \sup _{\|x\|_{\infty} \geq M} P\left(x \in R\left(X_{1}, X_{2}, \ldots, X_{j}\right)\right) \\
& \leq \sum_{j=2}^{J} \sup _{\|x\|_{\infty} \geq M} \sum_{r=1}^{j} P\left(\left\|X_{r}\right\|_{\infty} \geq\|x\|_{\infty}\right) \\
& \leq \sum_{j=2}^{J} \sum_{r=1}^{j} \sup _{\|x\|_{\infty} \geq M} P\left(\left\|X_{r}\right\|_{\infty} \geq\|x\|_{\infty}\right)
\end{aligned}
$$

and $\sup _{\|x\|_{\infty} \geq M} S_{J}(x) \longrightarrow 0$, when $M \rightarrow \infty$. To prove that the sample depth converges almost surely to zero we use again the inclusion in $(7) . S_{n}^{(j}(x)$ is bounded by

$$
\left(\begin{array}{c}
n \\
j
\end{array}\right)^{-1} \sum_{1 \leq i_{1}<\ldots i_{j} \leq n} I\left\{\bigcup_{r=1}^{j}\left\{\left\|X_{i_{r}}\right\|_{\infty} \geq\|x\|_{\infty}\right\}\right\},
$$

where $I\left\{\bigcup_{r=1}^{j}\left\{\left\|X_{i_{r}}\right\|_{\infty} \geq\|x\|_{\infty}\right\}\right\}$ is 1 if $\left\|X_{i_{r}}\right\|_{\infty} \geq\|x\|_{\infty}$ for some $r$ and 0 in any other case. Then,

$$
\begin{aligned}
\sup _{\|x\|_{\infty} \geq M} S_{n}^{(j}(x) & \leq \sup _{\|x\|_{\infty} \geq M}\left(\begin{array}{c}
n \\
j
\end{array}\right)^{-1} \sum_{1 \leq i_{1}<\ldots i_{j} \leq n} I\left\{\bigcup_{r=1}^{j}\left\{\left\|X_{i_{r}}\right\|_{\infty} \geq\|x\|_{\infty}\right\}\right\} \\
& \leq\left(\begin{array}{c}
n \\
j
\end{array}\right)^{-1} \sum_{1 \leq i_{1}<\ldots<i_{j} \leq n} \sum_{r=1}^{j} \sup _{\|x\|_{\infty} \geq M} I\left\{\left\|X_{i_{r}}\right\|_{\infty} \geq\|x\|_{\infty}\right\} .
\end{aligned}
$$

Next, we prove that $X_{M}=\sup _{\|x\|_{\infty} \geq M} I\left\{\left\|X_{i_{r}}\right\|_{\infty} \geq\|x\|_{\infty}\right\}$ converges almost surely to 0 when $M$ tends to infinity (for any $r$ ). Let $Y_{M}=I\left\{\left\|X_{i_{r}}\right\|_{\infty} \geq M\right\}$; since $0 \leq X_{M} \leq Y_{M}$, it is sufficient 
to prove that $Y_{M} \stackrel{a . s .}{\longrightarrow} 0$ or, equivalently, that $P\left(\sup _{M \geq l} I\left\{\left\|X_{i_{r}}\right\|_{\infty} \geq M\right\}>\varepsilon\right) \longrightarrow 0$, when $l \rightarrow \infty$. Since $\sup _{M \geq l} I\left\{\left\|X_{i_{r}}\right\|_{\infty} \geq M\right\} \leq I\left\{\left\|X_{i_{r}}\right\|_{\infty} \geq l\right\}, P\left(\sup _{M \geq l} I\left\{\left\|X_{i_{r}}\right\|_{\infty} \geq M\right\}>\varepsilon\right) \leq$ $P\left(I\left\{\left\|X_{i_{r}}\right\|_{\infty} \geq l\right\}>\varepsilon\right)=P\left(\left\|X_{i_{r}}\right\|_{\infty} \geq l\right) \longrightarrow 0$, when $l \longrightarrow \infty$. Therefore, $\sup _{\|x\|_{\infty} \geq M} S_{n}^{(j}(x) \stackrel{a . s .}{\longrightarrow}$ 0 , when $M \rightarrow \infty$ and $\sup _{\|x\|_{\infty} \geq M} S_{n, J}(x) \stackrel{a . s}{\longrightarrow} 0$, when $M \rightarrow \infty$.

(iv) $S^{(j}$ is an upper- semicontinuous function: if $x_{n} \rightarrow x$,

$$
\begin{aligned}
\limsup _{n \rightarrow \infty} S^{(j}\left(x_{n}\right) & =\limsup _{n \rightarrow \infty} P\left(x_{n} \in R\left(X_{1}, \ldots X_{j}\right)\right) \leq P\left(\limsup _{n \rightarrow \infty}\left\{x_{n} \in R\left(X_{1}, \ldots X_{j}\right)\right\}\right) \\
& \leq P\left(x \in R\left(X_{1}, \ldots, X_{j}\right)\right)=S^{(j}(x) .
\end{aligned}
$$

$S_{J}$ is also upper- semicontinuous:

$$
\limsup _{n \rightarrow \infty} S_{J}\left(x_{n}\right)=\limsup _{n \rightarrow \infty} \sum_{j=2}^{J} S^{(j}\left(x_{n}\right) \leq \sum_{j=2}^{J} \limsup _{n \rightarrow \infty} S^{(j}\left(x_{n}\right) \leq \sum_{j=2}^{J} S^{(j}(x)=S_{J}(x) .
$$

To establish that $S_{J}(x)$ is continuous under distributions $P$ with continuous marginals, it is enough to prove it for $S_{n}^{j)}(x)$. Assume that $x_{n}$ converges to $x$. Since

$$
\left\{x_{n} \in R\left(X_{1}, \ldots, X_{j}\right)\right\} \subset\left\{x \in R\left(X_{1}, \ldots, X_{j}\right)\right\} \cup\left\{x_{n} \in R\left(X_{1}, \ldots, X_{j}\right) \cap x \notin R\left(X_{1}, \ldots, X_{j}\right)\right\}
$$

it follows that

$$
\begin{aligned}
\left|P\left(x_{n} \in R\left(X_{1}, \ldots, X_{j}\right)\right)-P\left(x \in R\left(X_{1, \ldots,} X_{j}\right)\right)\right| & \leq P\left(x_{n} \in R\left(X_{1}, \ldots, X_{j}\right) \cap x \notin R\left(X_{1}, \ldots, X_{j}\right)\right)+ \\
& +P\left(x_{n} \notin R\left(X_{1}, \ldots, X_{j}\right) \cap x \in R\left(X_{1}, \ldots, X_{j}\right)\right) .
\end{aligned}
$$

Therefore,

$$
\begin{aligned}
\left|S^{(j}\left(x_{n}\right)-S^{(j}(x)\right|= & \left|P\left(x_{n} \in R\left(X_{1}, \ldots, X_{j}\right)\right)-P\left(x \in R\left(X_{1}, \ldots, X_{j}\right)\right)\right| \\
\leq & P\left(x_{n} \in R\left(X_{1}, \ldots, X_{j}\right), x \notin R\left(X_{1}, \ldots, X_{j}\right)\right)+ \\
& +P\left(x_{n} \notin R\left(X_{1}, \ldots, X_{j}\right), x \in R\left(X_{1}, \ldots, X_{j}\right)\right) \leq 2 P\left(A_{n}\right),
\end{aligned}
$$

where $A_{n}=\bigcup_{i=1}^{j} \bigcup_{k=1}^{d}\left\{\min \left\{x(k), x_{n}(k)\right\} \leq X_{i}(k) \leq \max \left\{x(k), x_{n}(k)\right\}\right\}$ and, since the marginals of $P$ are absolutely continuous, $P\left(A_{n}\right)$ converges to zero when $x_{n} \rightarrow x$. 
Proof of Proposition 1: $S^{(j}(x)$ is a $U$-statistic with kernel $h_{j}\left(x_{1}, x_{2}, \ldots, x_{j}\right)=I\left\{x \in R\left(x_{1}, x_{2}, \ldots, x_{j}\right)\right\}$ (see, e.g., Serfling, 1980). We prove that the sum of $U$-statistics of orders $\{2, \ldots, J\}$ is a $U$ statistic of order $J$. Let $H_{J}=\sum_{j=2}^{J} H^{j)}\left(x_{1}, \ldots, x_{n}\right)$ with

$$
H^{j)}\left(x_{1}, \ldots, x_{n}\right)=\left(\begin{array}{c}
n \\
j
\end{array}\right)^{-1} \sum_{1 \leq i_{1}<\ldots<i_{j} \leq n} h_{j}\left(x_{i_{1}}, \ldots, x_{i_{j}}\right) .
$$

We have that

$H_{J}=\frac{\frac{\left(\begin{array}{l}n \\ J\end{array}\right)}{\left(\begin{array}{l}n \\ 2\end{array}\right)} \sum_{1 \leq i_{1}<i_{2} \leq n} h_{2}\left(x_{i_{1}}, x_{i_{2}}\right)+\ldots+\frac{\left(\begin{array}{l}n \\ J\end{array}\right)}{\left(\begin{array}{l}n \\ j\end{array}\right)} \sum_{1 \leq i_{1}<\ldots<i_{j} \leq n} h_{j}\left(x_{i_{1}}, \ldots, x_{i_{j}}\right)+\ldots+\sum_{1 \leq i_{1}<\ldots<i_{J} \leq n} h_{J}\left(x_{i_{1}}, \ldots, x_{i_{J}}\right)}{\left(\begin{array}{l}n \\ J\end{array}\right)}$,

and, in general, the coefficient of the $j$-th term in $H_{J}$ is $\frac{\left(\begin{array}{l}n \\ J\end{array}\right)}{\left(\begin{array}{c}n \\ j\end{array}\right)}=\frac{j !(n-j) !}{J !(n-J) !}$. On the other hand, consider the projection $h^{p j}: \mathbb{R}^{J} \rightarrow \mathbb{R}$ given by $h^{p j}\left(x_{1}, \ldots, x_{J}\right)=h_{j}\left(x_{1}, \ldots, x_{j}\right)$.

The symmetrized version of $h^{p j}$ is $h^{* j}\left(x_{1}, \ldots, x_{J}\right)=\frac{\sum_{*} h^{p j}\left(x_{\sigma(1)}, \ldots, x_{\sigma(J)}\right)}{J !}$, where the sum is over all permutations of indexes $1, \ldots, J$. The function $h^{* j}$ is a symmetric function defined on $\mathbb{R}^{J}$. It holds that

$$
\sum_{1 \leq i_{1}<i_{2}<\ldots<i_{J} \leq n} h^{* j}\left(x_{i_{1}}, \ldots, x_{i_{J}}\right)=\frac{\left(\begin{array}{c}
n-j \\
J-j
\end{array}\right) j !(J-j) ! \sum_{1 \leq i_{1}<i_{2}<\ldots<i_{j} \leq n} h_{j}\left(x_{i_{1}}, \ldots, x_{i_{j}}\right)}{J !}
$$

and

$$
\sum_{1 \leq i_{1}<i_{2}<\ldots<i_{j} \leq n} h_{j}\left(x_{i_{1}}, \ldots, x_{i_{j}}\right)=\frac{J !}{\left(\begin{array}{c}
n-j \\
J-j
\end{array}\right) j !(J-j) !} \sum_{1 \leq i_{1}<i_{2}<\ldots<i_{J} \leq n} h^{* j}\left(x_{i_{1}}, \ldots, x_{i_{J}}\right) .
$$

Plugging (13) in equation (11), we obtain

$$
H_{J}=\frac{\sum_{1 \leq i_{1}<\ldots<i_{J} \leq n} h^{* 2}\left(x_{i_{1}}, \ldots, x_{i_{J}}\right)+\ldots \sum_{1 \leq i_{1}<\ldots<i_{J} \leq n} h_{J}\left(x_{i_{1}}, \ldots, x_{i_{J}}\right)}{\left(\begin{array}{l}
n \\
J
\end{array}\right)} .
$$

Defining the symmetric kernel $h^{\text {def }}: \mathbb{R}^{J} \rightarrow \mathbb{R}$ as

$$
h^{d e f}\left(x_{1}, \ldots, x_{J}\right)=h^{* 2}\left(x_{1}, \ldots, x_{J}\right)+\ldots+h^{* j}\left(x_{1}, \ldots, x_{J}\right)+\ldots+h_{J}\left(x_{1}, \ldots, x_{J}\right),
$$

$H_{J}$ is a $U$-statistic of order $J$ with kernel $h^{\text {def }}$,

$$
H_{J}\left(x_{1}, \ldots, x_{n}\right)=\frac{\sum_{1 \leq i_{1}<i_{2}<\ldots<i_{J} \leq n} h^{\operatorname{def}}\left(x_{i_{1}}, \ldots, x_{i_{J}}\right)}{\left(\begin{array}{l}
n \\
J
\end{array}\right)} .
$$


The expected value of $H_{J}$ is

$$
\begin{aligned}
E^{J}\left(H_{J}\right) & =\frac{\sum_{1 \leq i_{1}<i_{2}<\ldots<i_{J} \leq n} E^{J}\left(h^{\operatorname{def}}\left(X_{i_{1}}, \ldots, X_{i_{J}}\right)\right)}{\left(\begin{array}{l}
n \\
J
\end{array}\right)}=E^{J}\left(h^{\operatorname{def}}\left(X_{1}, \ldots, X_{J}\right)\right) \\
& =E^{J}\left(h^{* 2}\left(X_{1}, \ldots, X_{J}\right)\right)+\ldots+E^{J}\left(h^{* j}\left(X_{1}, \ldots, X_{J}\right)\right)+\ldots+E^{J}\left(h_{J}\left(X_{1}, \ldots, X_{J}\right)\right)
\end{aligned}
$$

and since the sample is identically distributed the expectation of a general term is

$$
E^{J}\left(h^{* j}\left(X_{1}, \ldots, X_{J}\right)\right)=E^{J}\left(h^{p j}\left(X_{1}, \ldots, X_{J}\right)\right)=E^{J}\left(h_{j}\left(X_{1}, \ldots, X_{j}\right)\right)=E^{j}\left(h_{j}\left(X_{1}, \ldots, X_{j}\right)\right)
$$

Then $E^{J}\left(H_{J}\right)=E^{2}\left(h_{2}\left(X_{1}, X_{2}\right)\right)+\ldots+E^{J}\left(h_{J}\left(X_{1}, \ldots, X_{J}\right)\right)$.

Proof of Proposition 2: Assume that $P$ is antipodally symmetric about zero (i.e., $X$ and $-X$ are equally distributed). Let $X_{s}=\left\{X_{1}, \ldots, X_{n}\right\}$ be a random sample from $P$ and let $\widehat{m}_{n, J}\left(X_{s}\right)$ be the sample median based on $S_{n, J}$. Due to invariance under $T(X)=A X+b$, where $A$ is a diagonal and invertible matrix, it can be shown that $\widehat{m}_{n, J}\left(-X_{s}\right)=-\widehat{m}_{n, J}\left(X_{s}\right)$, where $-X_{s}=-X_{1}, \ldots,-X_{n}$. Since $X$ and $-X$ are identically distributed, $\widehat{m}_{n, J}\left(-X_{s}\right)$ and $\widehat{m}_{n, J}\left(X_{s}\right)$ are also identically distributed; hence, $\widehat{m}_{n, J}\left(X_{s}\right)$ and $-\widehat{m}_{n, J}\left(X_{s}\right)$ are identically distributed and $\widehat{m}_{n, J}(X)$ is symmetric around zero.

Proof of Theorem 2: (i) By triangular inequality,

$$
\sup _{x \in R^{d}}\left|S_{n, J}(x)-S_{J}(x)\right| \leq \sup _{x \in R^{d}}\left|\sum_{j=2}^{J}\left(S_{n}^{(j}(x)-S^{(j}(x)\right)\right| \leq \sum_{j=2}^{J} \sup _{x \in R^{d}}\left|S_{n}^{(j}(x)-S^{(j}(x)\right|
$$

and it is enough to show that

$$
\sup _{x \in R^{d}}\left|S_{n}^{(j}(x)-S^{(j}(x)\right| \stackrel{a . s .}{\longrightarrow} 0, \text { when } n \rightarrow \infty .
$$

Since $S_{n}^{(j}(\cdot)$ is a $U$-process, we can use the results in Arcones and Giné (1993) establishing that if the family of functions $\mathcal{F}$ is $\mathrm{VC}$ with envelope $G$ and $P^{m} G<\infty$ then $\left\|U_{m}^{n}-P^{m}\right\|_{\mathcal{F}} \stackrel{\text { a.s. }}{\longrightarrow} 0$. In this case $\mathcal{F}=\left\{R_{x}: x \in \mathbb{R}^{d}\right\}$ is a VC class of functions (see Corollary 6.7 in Arcones and Giné, 1993) and the envelope $G$ is the function identically equal to one in its domain $\left(\mathbb{R}^{d}\right)^{j}$. The second part follows by adapting Theorem 6.9 in Arcones and Giné (1993) to the band depth $S_{J}$. (iii) follows as the second part of Theorem 5 in Liu (1990) since $S_{J}(\cdot)$ verifies that theorem conditions. 
Proof of Proposition 3: We have that

$$
\begin{aligned}
S^{(j}\left(x_{m}\right) & =P\left(\min _{i=1, \ldots, j}\left\{X_{m, i}\left(q_{k}\right)\right\} \leq x_{m}\left(q_{k}\right) \leq \max _{i=1, \ldots, j}\left\{X_{m, i}\left(q_{k}\right)\right\}, k=1, \ldots, m\right)= \\
& =P\left(\bigcap_{k=1}^{m}\left\{\min _{i=1, \ldots, j}\left\{X_{m, i}\left(q_{k}\right)\right\} \leq x_{m}\left(q_{k}\right) \leq \max _{i=1, \ldots, j}\left\{X_{m, i}\left(q_{k}\right)\right\}\right\}\right) .
\end{aligned}
$$

Let $A_{m}=\bigcap_{k=1}^{m}\left\{\min _{i=1, \ldots, j}\left\{X_{m, i}\left(q_{k}\right)\right\} \leq x_{m}\left(q_{k}\right) \leq \max _{i=1, \ldots, j}\left\{X_{m, i}\left(q_{k}\right)\right\}\right\}$. Thus $A_{m+1} \subset A_{m}$ for each $m$ and $\lim _{m \rightarrow \infty} P\left(A_{m}\right)=P\left(\bigcap_{m=1}^{\infty} A_{m}\right)$. Hence,

$$
\begin{aligned}
\lim _{m \rightarrow \infty} S^{(j}\left(x_{m}\right) & =P\left(\bigcap_{k=1}^{\infty}\left\{\min _{i=1, \ldots, j}\left\{X_{i}\left(q_{k}\right)\right\} \leq x\left(q_{k}\right) \leq \max _{i=1, \ldots, j}\left\{X_{i}\left(q_{k}\right)\right\}\right\}\right) \\
& =P\left(\min _{i=1, \ldots, j}\left\{X_{i}(t)\right\} \leq x(t) \leq \max _{i=1, \ldots, j}\left\{X_{i}(t)\right\}, t \in I\right)=S^{(j}(x),
\end{aligned}
$$

what implies that $\lim _{m \rightarrow \infty} S_{J}\left(x_{m}\right)=S_{J}(x)$.

Proof of Theorem 3: (i) follows from the definition. The proof of (ii) is analogous to the finite-dimensional case. (iii) $S_{J}$ is upper-semicontinuous (following the finite-dimensional argument). For $S_{J}$ continuity, we express it in terms of the rational numbers in $I$,

$$
S^{(j}(x)=P\left(\min _{i=1, \ldots, j}\left\{X_{i}(q)\right\} \leq x(q) \leq \max _{i=1, \ldots, j}\left\{X_{i}(q)\right\}, \quad q \in \mathbb{Q} \cap I\right) .
$$

For any sequence $\left\{x_{n}\right\}$ in $C(I)$ such that $x_{n} \stackrel{\|\cdot\|_{\infty}}{\longrightarrow} x$,

$$
\begin{aligned}
\left|S^{(j}\left(x_{n}\right)-S^{(j}(x)\right|= & \left|P\left(G\left(x_{n}\right) \subset V\left(X_{1}, \ldots, X_{j}\right)\right)-P\left(G(x) \subset R\left(X_{1}, \ldots, X_{j}\right)\right)\right| \\
\leq & P\left(G\left(x_{n}\right) \subset V\left(X_{1}, \ldots, X_{j}\right), G(x) \varsubsetneqq V\left(X_{1}, \ldots, X_{j}\right)\right)+ \\
& +P\left(G\left(x_{n}\right) \varsubsetneqq V\left(X_{1}, \ldots, X_{j}\right), G(x) \subset V\left(X_{1}, \ldots, X_{j}\right)\right) \\
\leq & 2 P\left(A_{n}\right),
\end{aligned}
$$

where

$$
A_{n}=\bigcup_{i=1}^{j} \bigcup_{q \in \mathbb{Q} \cap I}\left\{\min \left\{x(q), x_{n}(q)\right\} \leq X_{i}(q) \leq \max \left\{x(q), x_{n}(q)\right\}\right\} .
$$

If the marginal distributions of $P$ are absolutely continuous, then, for $x \in C(I)$,

$$
P\left(\bigcup_{q \in \mathbb{Q} \cap I}\{X(q)=x(q)\}\right)=0 .
$$

Therefore, $P\left(A_{n}\right)$ converges to zero when $n$ tends to infinity, what implies that $S^{(j}$ is continuous and this is inherited by $S_{J}$. 
Proof of Theorem 4: Without loss of generality, consider $I=[0,1]$. We have that

$$
\sup _{x \in E}\left|S_{n, J}(x)-S_{J}(x)\right| \leq \sup _{x \in E,\|x\|_{\infty}<M}\left|S_{n, J}(x)-S_{J}(x)\right|+\sup _{x \in E,\|x\|_{\infty} \geq M}\left|S_{n, J}(x)-S_{J}(x)\right| .
$$

Due to (ii) in Theorem 3, the second term converges to zero when $M$ tends to infinity. Therefore, it is sufficient to prove that for $M$ large enough, the first term converges to zero when $n \rightarrow \infty$. By Ascoli-Arzela's Theorem (see, e.g., Pollard, 1984), the closure $\bar{E}_{M}$ of $E_{M}=\left\{x \in E:\|x\|_{\infty}<M\right\}$ is compact and, hence, totally bounded. Then, for every $\varepsilon>0$, there exists a finite set $\left\{x_{1}, x_{2}, \ldots, x_{N_{\varepsilon}}\right\}$ of functions such that $\bar{E}_{M} \subseteq \bigcup_{m=1}^{\infty} B\left(x_{m}, \varepsilon\right)$. By Theorem 3. (iii), to establish that $\sup \left|S_{n, J}(x)-S_{J}(x)\right| \stackrel{\text { a.s. }}{\longrightarrow} 0$, when $n \rightarrow \infty$, it is enough to prove $\max _{x \in\left\{x_{m}\right\}_{m=1}^{N_{\varepsilon}}}\left|S_{n, J}(x)-S_{J}(x)\right| \stackrel{x \in \bar{E}_{M}}{\stackrel{a . s .}{\longrightarrow}} 0$, when $n \rightarrow \infty$. But, we have that

$$
\begin{aligned}
P\left\{\max _{x \in\left\{x_{m}\right\}_{m=1}^{N_{\varepsilon}}}\left|S_{n, J}(x)-S_{J}(x)\right| \geq \epsilon\right\} & \leq N_{\varepsilon} \max _{x \in\left\{x_{m}\right\}_{m=1}^{N_{\varepsilon}}} P\left\{\left|S_{n, J}(x)-S_{J}(x)\right| \geq \epsilon\right\} \\
& \leq N_{\varepsilon} \max _{x \in\left\{x_{m}\right\}_{m=1}^{N_{\varepsilon}}} \frac{E\left[\left|S_{n, J}(x)-S_{J}(x)\right|^{4}\right]}{\varepsilon^{4}}=O\left(n^{-2}\right),
\end{aligned}
$$

and Borel-Cantelli's Lemma provides the result in (i). To prove (ii), we use that $m_{n} \stackrel{\text { a.s. }}{\longrightarrow} m$ if, and only if, $P\left(\sup _{n \geq l}\left\|m_{n}-m\right\|_{\infty} \geq \varepsilon\right) \underset{l \rightarrow \infty}{\longrightarrow} 0 .\left(E,\|\cdot\|_{\infty}\right)$ is a metric space and $S_{J}(\cdot)$ is an upper-semicontinuous function in $E$ and verifies that $\sup _{\|x\|_{\infty} \geq M, x \in E} S_{J}(x) \underset{M \rightarrow \infty}{\longrightarrow} 0$; thus, the proof is analogous to that of Theorem 2 (ii).

\section{REFERENCES}

Arcones, M.A. and Giné, E. (1993), Limit theorems for U-processes, The Annals of Probability, 21, 1494-1542.

Brown, B. and Hettmansperger, T. (1989), The affine invariant bivariate version of the sign test, Journal of the Royal Statistical Society B, 51, 117-125.

Fraiman, R. and Meloche, J. (1999), Multivariate L-estimation, Test, 8, 255-317.

Fraiman, R. and Muniz, G. (2001), Trimmed means for functional data, Test, 10, 419-440. 
Hettmansperger, T. and Oja, H. (1994), Affine invariant multivariate multisample sign tests, Journal of the Royal Statistical Society B, 56, 235-249.

Inselberg, A. (1981), N-dimensional graphics, Part I-lines and hyperplanes, in IBM LASC, Technical Report, G320-27111.

Inselberg, A. (1985), The plane parallel coordinates, Invited paper, Visual Computer, 1, 69-91.

Inselberg, A., Reif, M. y Chomut, T. (1987), Convexity algorithms in parallel coordinates, Journal of ACM, 34, 765-801.

Li, J. and Liu, R. (2004), New Nonparametric Tests of Multivariate Locations and Scales using Data Depth, Statistical Science, 19, 4, 686-696.

Liu, R. (1990), On a notion of data depth based on random simplices, The Annals of Statistics, 18, 405-414.

Liu, R. (1995), Control charts for multivariate processes, Journal of the American Statistical Association, 90, 1380-1388.

Liu, R., Parelius, J.M. and Singh, K. (1999), Multivariate analysis by data depth: Descriptive statistics, graphics and inference, Annals of Statistics, 27, 783-858.

Liu, R. and Singh, K. (1993), A quality index based on data depth and multivariate rank test, Journal of the American Statistical Association, 88, 257-260.

Mahalanobis, P. C. (1936), On the generalized distance in statistics, Proceedings of National Academy of Science of India, 12, 49-55.

Oja, H. (1983), Descriptive statistics for multivariate distributions, Statistics and Probability Letters, 1, 327-332.

Pollard, D. (1984), Convergence of stochastic processes. Springer Verlag, New York.

Ramsay, J.O. and Silverman, B.W. (2005), Functional Data Analysis. Second Edition. 
Springer Verlag, New York.

Rousseeuw, P. and Hubert, M. (1999), Regression depth (with discussion), Journal of the American Statistical Association, 4, 388-433.

Serfling, R.J. (1980), Approximation Theorems of Mathematical Statistics, Wiley, New York.

Singh, K. (1991), A notion of majority depth, Unpublished document.

Tukey, J. (1975), Mathematics and picturing data, Proceedings of the $19^{75}$ International Congress of Mathematics, 2, 523-531.

Vardi, Y. and Zhang, C.-H.(2001), The multivariate L1-median and associated data depth, Proceedings of the National Academy of Science USA, 97, 1423-1426.

Wegman, E. (1990), Hyperdimensional data analysis using parallel coordinates, Journal of the American Statistical Association, 85, 664-675.

Wood, A.T.A. and Chan, G. (1994), Simulation of stationary gaussian processes in $C[0,1]$, Journal of Computational and Graphical Statistics, 3, 409-432.

Yeh, A. and Singh, K. (1997), Balanced confidence sets based on the Tukey depth, Journal of the Royal Statistical Society Series B, 3, 639-652.

Zuo, Y. (2003), Projection based depth functions and associated medians, The Annals of Statistics, 31, 1460-1490.

Zuo, Y. and Serfling, R. (2000), General notions of statistical depth function, The Annals of Statistics, 28, 461-482. 